\title{
Demonstrating the Synergy of Synthetic, Mechanistic, and Computational Studies in a Regioselective Aniline Synthesis.
}

Ian W. Davies,* Jean-François Marcoux, Jeffery T. Kuethe, Michael D. Lankshear, Jeremy D.O. Taylor, Nancy Tsou, Peter G. Dormer and David L. Hughes. Department of Process Research, Merck \& Co., Inc., PO Box 2000, Rahway, NJ 07065-0900.

K. N. Houk and Vildan Guner. Department of Chemistry and Biochemistry, University of California, Los Angeles, 405 Hilgard Avenue, Los Angeles, CA 90095-1569.

\section{Table of Contents}

Scheme S1:

Formulae for calculation of kinetic and product isotope effects. 3

Experimental Section

Characterization data for all new compounds

General procedure for the determination of isotope effects

Supporting Information for X-ray analysis of 21d

Table 1. Summary of Structure Determination of 21d

Table 2. Bond Distances in Angstroms

Table 3. Bond Angles in Degrees

Table 4. Fractional Atomic Coordinates and Equivalent Isotropic Displacement Parameters

Table 5. Anisotropic Displacement Parameters.

Cartesian coordinates of reactants, transition structures, and products at B3LYP/6-31G* 
R49:1,3-Cyclohexadiene and TS50( transition structure)

R27: 1-Hydroxy-2-methylacetate-4-nitro-5-N-dimethylamine-1,3-cyclohexadiene and TS-51

R30: 1-N-dimethylamine-2-nitro-4-mehylacetate-5-hydroxy-1,3-cyclohexadiene.

Cartesian coordinates of reactants, transition structures at B3LYP/6-31G* level for Electrocyclic ring closure. Total energies (au) are given.

R52: $1,3,5-$ Hexatriene and TS

R26: Reactant with substituent $\mathrm{NO}_{2}$ ( for enol case) and TS

R28: Reactant with substituent $\mathrm{NO}_{2}$ ( for enamine case) and TS

R58: Reactant with substituent $\mathrm{C}=\mathrm{N}^{+} \mathrm{Me}_{2}$ ( for enol case) and TS-59

R55: Reactant with substituent $\mathrm{C}=\mathrm{N}^{+} \mathrm{Me}_{2}$ ( for enamine case) and TS-56 


\section{KIE/PIE Experiments}

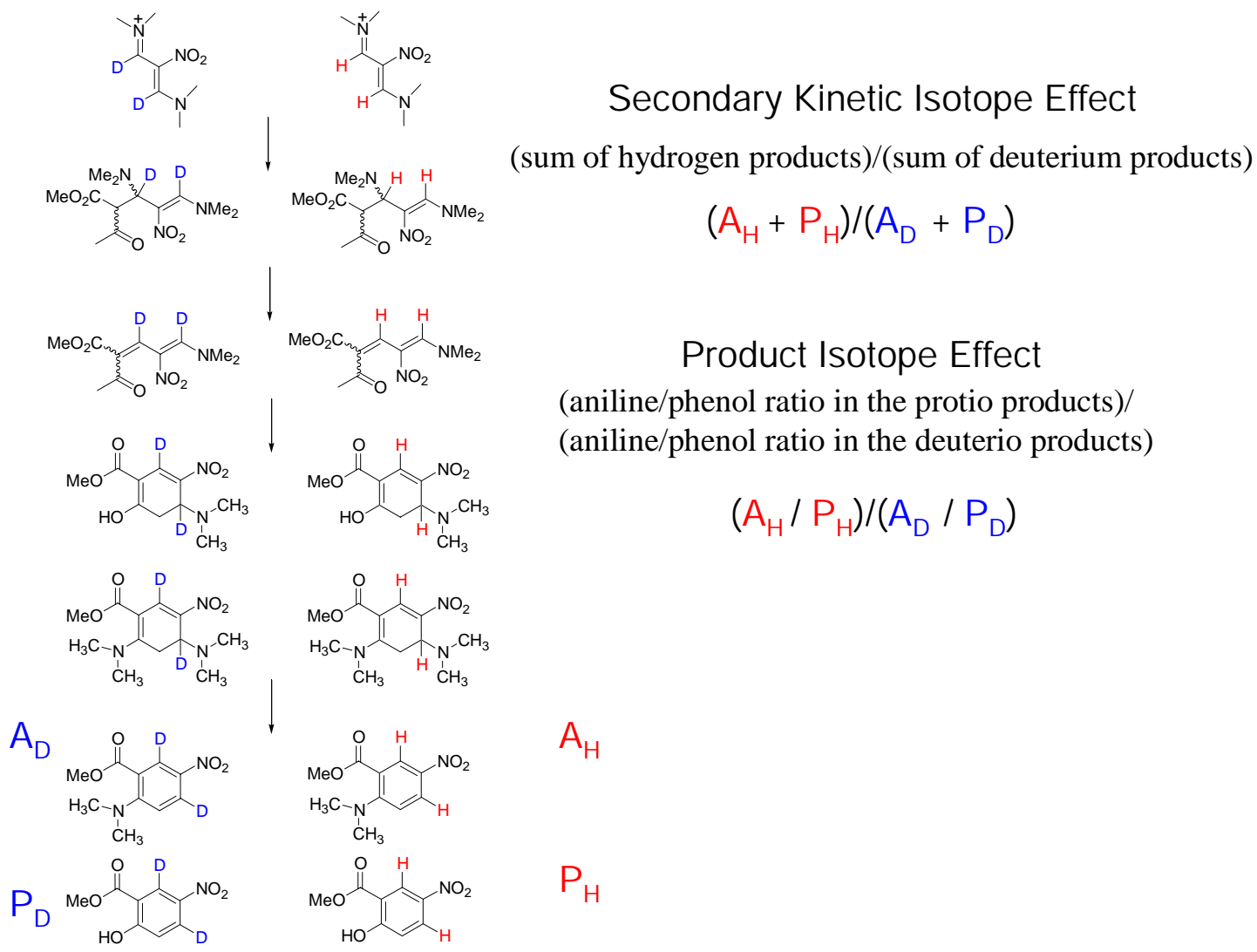

Scheme S1: Roadmap for isotopic distribution and formulae for calculation of kinetic and product isotope effects. 


\section{Experimental Section}

General procedure for the formation of anilines is exemplified by the preparation of 4 :

\section{Benzoic acid, 6-(dimethylamino)-3-nitro-, methyl ester $4^{1}$}

To a stirred solution of methylacetoacetate $(580 \mathrm{mg}, 5 \mathrm{mmol})$ in acetonitrile $(10 \mathrm{~mL})$ at $-10{ }^{\circ} \mathrm{C}$ was added potassium tert-butoxide $(5.25 \mathrm{~mL}, 1.0 \mathrm{M}$ in acetonitrile) over 5 minutes. The solution was aged for 5 minutes and nitrovinamidinium hexafluorophosphate $3 \mathrm{~g}$ (1.74 g, $5.5 \mathrm{mmol})$ was added in one portion in acetonitrile $(5 \mathrm{~mL})$. The mixture was stirred at $-10{ }^{\circ} \mathrm{C}$ for $12 \mathrm{hr}$. The mixture was partitioned between ethylacetate and water. Concentration of the organic extracts and chromatography on silica gel eluting with ethylacetate/heptane gave aniline $4(876 \mathrm{mg}, 78 \%)$ as a light yellow solid. DSC peak 88.4 ${ }^{\circ} \mathrm{C}$ (lit. $\left.71.5^{\circ} \mathrm{C}\right) .{ }^{1} \mathrm{H} \mathrm{NMR}\left(400 \mathrm{MHz}, \mathrm{CDCl}_{3}\right) \delta 8.55(1 \mathrm{H}, \mathrm{d}, J=3 \mathrm{~Hz}), 8.15(1 \mathrm{H}, \mathrm{dd}, J=9,3 \mathrm{~Hz}), 6.85$ $(1 \mathrm{H}, \mathrm{d}, J=9 \mathrm{~Hz}), 3.93(3 \mathrm{H}, \mathrm{s}), 3.03(6 \mathrm{H}, \mathrm{s}) .{ }^{13} \mathrm{C} \mathrm{NMR}\left(100 \mathrm{MHz}, \mathrm{CDCl}_{3}\right) \delta$ 167.1, 155.0, 137.1, 128.9, 127.4, 116.6, 114.6, 52.5, 42.9. m/z [M+H] $]^{+}$225.0864. Found: C, 53.66; H, 5.39; N, 12.30. $\mathrm{C}_{10} \mathrm{H}_{12} \mathrm{~N}_{2} \mathrm{O}_{4}$ requires $\mathrm{C}, 53.57 ; \mathrm{H}, 5.39 ; \mathrm{N}, 12.49$.

\section{Benzoic acid, 2-hydroxy-5-nitro-, methyl ester $5^{2}$}

${ }^{1} \mathrm{H}$ NMR $\left(400 \mathrm{MHz} \mathrm{CDCl}_{3}\right) \delta 11.44(1 \mathrm{H}, \mathrm{s}), 8.80(1 \mathrm{H}, \mathrm{d}, J=3 \mathrm{~Hz}), 8.34(1 \mathrm{H}, \mathrm{dd}, J=9,3 \mathrm{~Hz}), 7.10(1 \mathrm{H}$, $\mathrm{d}, J=9 \mathrm{~Hz}), 4.04(3 \mathrm{H}, \mathrm{s}) .{ }^{13} \mathrm{C} \mathrm{NMR}\left(100 \mathrm{MHz} \mathrm{CDCl}_{3}\right) \delta 169.3,166.2,140.0,130.5,126.6,118.6,112.1$, 53.1. Found: $\mathrm{C}, 49.05 ; \mathrm{H}, 3.54 ; \mathrm{N}, 6.76 . \mathrm{C}_{8} \mathrm{H}_{7} \mathrm{~N}_{2} \mathrm{O}_{5}$ requires, $\mathrm{C}, 48.74 ; \mathrm{H}, 3.58 ; \mathrm{N}, 7.10$.

\section{5-Benzenesulfonyl-2-hydroxy-benzoic acid methyl ester 11}

${ }^{1} \mathrm{H}$ NMR (400 MHz, $\left.\mathrm{CDCl}_{3}\right) \delta 11.29(1 \mathrm{H}, \mathrm{s}), 8.51(1 \mathrm{H}, \mathrm{s}), 8.00-7.90(3 \mathrm{H}, \mathrm{m}), 7.55-7.49(1 \mathrm{H}, \mathrm{m})$, $7.50-7.40(2 \mathrm{H}, \mathrm{m}), 7.05(1 \mathrm{H}, \mathrm{d}, J=8), 4.01(3 \mathrm{H}, \mathrm{s}) .{ }^{13} \mathrm{C} \mathrm{NMR}\left(100 \mathrm{MHz} \mathrm{CDCl}_{3}\right) \delta 169.4,165.1$, $141.8,134.5,133.1,132.3,130.8,129.3,127.4,119.0,112.7,52.9$. LCMS $[\mathrm{M}+\mathrm{H}]^{+}$293.0 Anal. Calcd. For $\mathrm{C}_{14} \mathrm{H}_{12} \mathrm{O}_{5} \mathrm{~S}: \mathrm{C}, 57.52 ; \mathrm{H}, 4.14$. Found: C, 57.10; H, 3.86.

\section{3-Benzenesulfonyl-4-dimethylamino-benzoic acid methyl ester 12}

Isolated as a light yellow oil. ${ }^{1} \mathrm{H} \mathrm{NMR}\left(400 \mathrm{MHz}_{\mathrm{CDCl}}\right) \delta 8.21(1 \mathrm{H}, \mathrm{d}, J=2 \mathrm{~Hz}), 7.92(2 \mathrm{H}, \mathrm{dd}, J=8$, $2 \mathrm{~Hz}), 7.78(2 \mathrm{H}, \mathrm{dd}, 9,2 \mathrm{~Hz}), 7.56-7.48(3 \mathrm{H}, \mathrm{m}), 6.89(1 \mathrm{H}, \mathrm{d}, J=9 \mathrm{~Hz}), 3.91(3 \mathrm{H}, \mathrm{s}), 2.96(6 \mathrm{H}, \mathrm{s}) ;{ }^{13} \mathrm{C}$ 
NMR $\left(100 \mathrm{MHz} \mathrm{CDCl}_{3}\right) \delta 167.4,154.1,132.5,132.3,133.1,130.0,129.8,129.1,127.9,127.1,117.6$,

115.3, 52.3, 42.7, 36.0. LCMS M+H 320.0.HRMS $[\mathrm{M}+\mathrm{H}]^{+}$320.0955. $\mathrm{C}_{16} \mathrm{H}_{18} \mathrm{NO}_{4} \mathrm{~S}$ requires 320.0951.

\section{Methanaminium,N,N'-[2-[(dimethylamino)methylene]-1,3-propanediylidene],[N-methyl-,}

\section{[tetrafluoroborate(1-)] [hexafluorophosphate(1-)] 13}

To a solution of trifluoropropanoic acid $(5.3 \mathrm{~g}, 0.041 \mathrm{~mol})$ in $\mathrm{DMF}(50 \mathrm{~mL})$ at $60{ }^{\circ} \mathrm{C}$ was added $\mathrm{POCl}_{3}$ over 15 minutes maintaining the temperature below $65^{\circ} \mathrm{C}$. After the addition was complete the mixture was heated to $75^{\circ} \mathrm{C}$ and $\mathrm{POCl}_{3}(6 \mathrm{~mL}, 0.05 \mathrm{~mol})$ was added maintaining the temperature below $85^{\circ} \mathrm{C}$. The mixture was heated for $6 \mathrm{hr}$ and cooled. The dark red DMF reaction mixture was added to a solution sodium tetrafluoroborate $(7.0 \mathrm{~g})$ in water $(100 \mathrm{~mL})$ maintaining the temperature below $15{ }^{\circ} \mathrm{C}$. The slurry was aged for $30 \mathrm{~min}$ at $5-10^{\circ} \mathrm{C}$ and then filtered washing with water/DMF (5:1) and dried to give $\mathbf{1 3}$ as an off-white solid in $43 \%$ yield. Colorless solid: DSC, peak $216.0{ }^{\circ} \mathrm{C} ;{ }^{1} \mathrm{H}$ NMR (400 $\left.\mathrm{MHz}, \mathrm{CD}_{3} \mathrm{CN}\right) \delta 8.05(1 \mathrm{H}, \mathrm{s}), 3.51(3 \mathrm{H}, \mathrm{s}), 2.89(3 \mathrm{H}, \mathrm{s}) ;{ }^{13} \mathrm{C} \mathrm{NMR}\left(100 \mathrm{MHz} \mathrm{CD}_{3} \mathrm{CN}\right) \delta 164.5,90.8$ 49.1, 43.2; ${ }^{19} \mathrm{~F}$ NMR (376 MHz, $\left.\mathrm{CD}_{3} \mathrm{CN}\right) \delta-73.1(\mathrm{~d}, J=709 \mathrm{~Hz}),-151.8$; Anal. Calcd. $\mathrm{C}_{10} \mathrm{H}_{21} \mathrm{BF}_{10} \mathrm{~N}_{3} \mathrm{P}: \mathrm{C}, 28.94 ; \mathrm{H}, 5.10, \mathrm{~N}, 10.12 ; \mathrm{B}, 2.60 ; \mathrm{P}, 7.46$. Found: C, 28.92; H, 4.94, N, 9.95; ICPAES analysis Found: B, 3.04; P, 6.96.

\section{Benzoic acid, 4-(dimethylamino)-3-formyl-, methyl ester 14}

Light yellow oil. ${ }^{1} \mathrm{H}$ NMR $\left(400 \mathrm{MHz}, \mathrm{CDCl}_{3}\right) \delta 9.74(1 \mathrm{H}, \mathrm{s}), 8.12(1 \mathrm{H}, \mathrm{s}), 7.70(1 \mathrm{H}, \mathrm{d}, J=8 \mathrm{~Hz}), 6.93$

$(1 \mathrm{H}, \mathrm{d}, J=8 \mathrm{~Hz}), 3.92(3 \mathrm{H}, \mathrm{s}), 3.01(6 \mathrm{H}, \mathrm{s}) ;{ }^{13} \mathrm{C} \mathrm{NMR}\left(100 \mathrm{MHz} \mathrm{CDCl}_{3}\right) \delta 189.6,168.0,155.2,135.8$ 132.3, 125.6, 117.4, 115.2, 52.2, 42.7. LCMS $[\mathrm{M}+\mathrm{H}]^{+}$208.1. Anal. Calcd. For $\mathrm{C}_{11} \mathrm{H}_{13} \mathrm{NO}_{2} \mathrm{C}, 65.76 ; \mathrm{H}$, 6.32; N, 6.76. Found: C, 65.53; H, 6.30; N, 6.61.

\section{4-Dimethylamino-3-(2-methoxycarbonyl-3-oxo-but-1-enyl)-benzoic acid methyl ester 16}

Isolated as a light yellow oil (1.5:1 mixture) $(5 \mathrm{mg})$. Minor isomer $(\mathrm{E}):{ }^{1} \mathrm{H} \mathrm{NMR}\left(400 \mathrm{MHz} \mathrm{CDCl}_{3}\right) \delta$ 7.81(1H, d, $J=2 \mathrm{~Hz}), 7.49(1 \mathrm{H}, \mathrm{s}), 7.42(1 \mathrm{H}, \mathrm{dd}, J=8,2 \mathrm{~Hz}), 6.80(1 \mathrm{H}, \mathrm{d}, J=8, \mathrm{~Hz}), 3.83(6 \mathrm{H}, \mathrm{s})$,

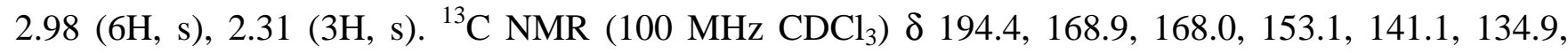


$133.3,130.5,120.9,118.3,52.2,52.1,42.8,31.2$. Major isomer $(\mathrm{Z}): \delta 7.81(1 \mathrm{H}, \mathrm{d}, J=2 \mathrm{~Hz}), 7.58(1 \mathrm{H}$,

s), 7.42(1H, dd, $J=8,2 \mathrm{~Hz}), 6.80(1 \mathrm{H}, \mathrm{d}, J=8, \mathrm{~Hz}), 3.90(6 \mathrm{H}, \mathrm{s}), 2.96(6 \mathrm{H}, \mathrm{s}), 2.35(3 \mathrm{H}, \mathrm{s}) .{ }^{13} \mathrm{C}$ NMR $\left(100 \mathrm{MHz}, \mathrm{CDCl}_{3}\right) \delta 203.9,168.1,165.3,152.8,140.4,135.2,132.9,129.7,121.3,118.5,52.3,52.2$, 42.8, 26.2. LCMS $[\mathrm{M}+\mathrm{H}]^{+}$306.0 HRMS $[\mathrm{M}+\mathrm{H}]^{+}$306.1348. $\mathrm{C}_{16} \mathrm{H}_{20} \mathrm{~N}_{2} \mathrm{O}_{5}$ requires 306.1336.

Benzoic acid, 4-(dimethylamino)-3-nitro-5-methyl, methyl ester 18a

Light yellow solid. ${ }^{1} \mathrm{H}$ NMR (400 $\left.\mathrm{MHz}, \mathrm{CDCl}_{3}\right) \delta 8.31(1 \mathrm{H}, \mathrm{d}, J=3 \mathrm{~Hz}), 8.11(1 \mathrm{H}, \mathrm{d}, J=3 \mathrm{~Hz}), 3.96$

$(3 \mathrm{H}, \mathrm{s}), 2.88(6 \mathrm{H}, \mathrm{s}), 2.39(3 \mathrm{H}, \mathrm{s}) ;{ }^{13} \mathrm{C} \mathrm{NMR}\left(100 \mathrm{MHz} \mathrm{CDCl}_{3}\right) \delta$ 167.5, 156.9, 141.0, 136.0, 128.7, 126.4, 124.6, 52.7, 42.9, 19.7; LCMS $[\mathrm{M}+\mathrm{H}]^{+}$239. HRMS $[\mathrm{M}+\mathrm{H}]^{+}$239.1010. $\mathrm{C}_{11} \mathrm{H}_{15} \mathrm{~N}_{2} \mathrm{O}_{4}$ requires 239.1032.

\section{4'-Fluoro-5-nitro-biphenyl-2-ol 19d}

Bright yellow solid: $\operatorname{mp} 83-84{ }^{\circ} \mathrm{C} ;{ }^{1} \mathrm{H}$ NMR $\left(\mathrm{CDCl}_{3}, 400 \mathrm{MHz}\right) \delta 2.71(\mathrm{~s}, 6 \mathrm{H}), 6.92(\mathrm{~d}, 1 \mathrm{H}, J=9.1$ $\mathrm{Hz}), 7.12(\mathrm{~m}, 2 \mathrm{H}), 7.45(\mathrm{~m}, 2 \mathrm{H}), 8.01(\mathrm{~d}, 1 \mathrm{H}, J=2.8 \mathrm{~Hz}), 8.08(\mathrm{dd}, 1 \mathrm{H}, J=9.1$ and $2.8 \mathrm{~Hz}) ;{ }^{13} \mathrm{C}$ NMR $\left(\mathrm{CDCl}_{3}, 100 \mathrm{MHz}\right) \delta 42.8,115.8(\mathrm{~d}, J=20 \mathrm{~Hz}), 116.1,124.3,128.1,129.9(\mathrm{~d}, J=10 \mathrm{~Hz}), 130.1,136.6$, 140.1, 156.2, $162.1(\mathrm{~d}, J=250 \mathrm{~Hz}) ;{ }^{19} \mathrm{~F} \mathrm{NMR}\left(\mathrm{CDCl}_{3}, 376 \mathrm{MHz}\right) \delta$ - 114.9. Anal. Calcd. For $\mathrm{C}_{14} \mathrm{H}_{13} \mathrm{FN}_{2} \mathrm{O}_{2}:$ C, 64.61; H, 5.03; N, 10.76. Found: C, 64.37; H, 4.93; N, 10.55. LCMS [M+H] ${ }^{+} 261.1$.

(3-(4-Chlorophenyl)-dimethylamino-2-nitro-allylidene)-dimethylammoniumhexafluorophosphate 20a

Yellow solid: mp. $181-182^{\circ} \mathrm{C} ;{ }^{1} \mathrm{H} \mathrm{NMR}\left(\mathrm{CDCl}_{3}, 400 \mathrm{MHz}\right) \delta 8.60(\mathrm{~s}, 1 \mathrm{H}), 7.74(\mathrm{~d}, 2 \mathrm{H}, \mathrm{J}=8.8 \mathrm{~Hz})$, $7.66(\mathrm{~d}, 2 \mathrm{H}, \mathrm{J}=8.8 \mathrm{~Hz}), 3.59(\mathrm{~s}, 3 \mathrm{H}), 3.50(\mathrm{~s}, 3 \mathrm{H}), 3.32(\mathrm{~s}, 3 \mathrm{H}), 2.67(\mathrm{~s}, 3 \mathrm{H}) ;{ }^{13} \mathrm{C} \mathrm{NMR}\left(\mathrm{CDCl}_{3} 100\right.$ MHz) $\delta 169.8,158.6,141.0,133.5,130.5,130.1,48.2,47.4,45.9,42.5 ;{ }^{19} \mathrm{~F} \mathrm{NMR}\left(\mathrm{CDCl}_{3} 376 \mathrm{MHz}\right) \delta-$ 73.1 (d, J = $706 \mathrm{~Hz}$ ). Anal. Calcd. For $\mathrm{C}_{13} \mathrm{H}_{17} \mathrm{ClF}_{6} \mathrm{~N}_{3} \mathrm{O}_{2} \mathrm{P}: \mathrm{C}, 36.51$; H, 4.01; N, 9.82. Found: C, 36.27; H, 3.75; N, 9.58.

(3-Dimethylamino-2-nitro-3-phenyl-allylidene)-dimethyl ammonium hexafluorophosphate 20b

Pale yellow solid: mp. $164-165^{\circ} \mathrm{C} ;{ }^{1} \mathrm{H} \mathrm{NMR}\left(\mathrm{CD}_{3} \mathrm{CN}, 400 \mathrm{MHz}\right) \delta 8.61(\mathrm{~s}, 1 \mathrm{H}), 7.77(\mathrm{~m}, 3 \mathrm{H}), 7.65(\mathrm{~m}$, 2H), $3.62(\mathrm{~s}, 3 \mathrm{H}), 3.51(\mathrm{~s}, 3 \mathrm{H}), 3.29(\mathrm{~s}, 3 \mathrm{H}), 2.64(\mathrm{~s}, 3 \mathrm{H}) ;{ }^{13} \mathrm{C} \mathrm{NMR}\left(\mathrm{CD}_{3} \mathrm{CN} 100 \mathrm{MHz}\right) \delta 158.5,135.1$, 
131.9, 131.8, 129.8, 117.3, 48.1, 47.3, 45.9, 42.4; ${ }^{19} \mathrm{~F}$ NMR $\left(\mathrm{CD}_{3} \mathrm{CN} 376 \mathrm{MHz}\right) \delta-73.2(\mathrm{~d}, \mathrm{~J}=707 \mathrm{~Hz})$.

Anal. Calcd. For $\mathrm{C}_{13} \mathrm{H}_{18} \mathrm{~F}_{6} \mathrm{~N}_{3} \mathrm{O}_{2} \mathrm{P}: \mathrm{C}, 39.70 ; \mathrm{H}, 4.61 ; \mathrm{N}, 10.68$. Found: C, 39.32; H, 4.33; N, 10.43.

(3-Dimethylamino-2-nitro-3-(4-methylphenyl)-allylidene)dimethylammoniumhexafluorophosphate 20c

Yellow solid: mp. $195-196{ }^{\circ} \mathrm{C} ;{ }^{1} \mathrm{H} \mathrm{NMR}\left(\mathrm{CD}_{3} \mathrm{CN}, 400 \mathrm{MHz}\right) \delta 8.59(\mathrm{~s}, 1 \mathrm{H}), 7.63(\mathrm{~d}, 2 \mathrm{H}, \mathrm{J}=8.3 \mathrm{~Hz})$, $7.45(\mathrm{~d}, 2 \mathrm{H}, \mathrm{J}=8.3 \mathrm{~Hz}), 3.60(\mathrm{~s}, 3 \mathrm{H}), 3.47(\mathrm{~s}, 3 \mathrm{H}), 3.26(\mathrm{~s}, 3 \mathrm{H}), 2.57(\mathrm{~s}, 3 \mathrm{H}), 2.47(\mathrm{~s}, 3 \mathrm{H}) ;{ }^{13} \mathrm{C}$ NMR $\left(\mathrm{CD}_{3} \mathrm{CN}, 100 \mathrm{MHz}\right) \delta 16.4,158.4,147.2,132.2,130.5,128.5,48.1,47.1,46.0,42.3,20.9 ; 19 \mathrm{~F}$ NMR $\left(\mathrm{CDCl}_{3}, 376 \mathrm{MHz}\right) \delta-73.4(\mathrm{~d}, \mathrm{~J}=707 \mathrm{~Hz})$. Anal. Calcd. For $\mathrm{C}_{13} \mathrm{H}_{20} \mathrm{~F}_{6} \mathrm{~N}_{3} \mathrm{O}_{2} \mathrm{P}: \mathrm{C}, 36.51 ; \mathrm{H}, 4.01 ; \mathrm{N}$, 9.82. Found: C, 36.27; H, 3.75; N, 9.58.

(3-Dimethylamino-3-(4-methoxy-phenyl)-2-nitro-allylidene)-dimethylammonium hexafluorophosphate 20d

Yellow solid: mp. $153-154^{\circ} \mathrm{C} ;{ }^{1} \mathrm{H} \mathrm{NMR}\left(\mathrm{CDCl}_{3}, 400 \mathrm{MHz}\right) \delta 8.62(\mathrm{~s}, 1 \mathrm{H}), 7.70(\mathrm{~d}, 2 \mathrm{H}, \mathrm{J}=8.9 \mathrm{~Hz})$, $7.13(\mathrm{~d}, 2 \mathrm{H}, \mathrm{J}=8.9 \mathrm{~Hz}), 3.92(\mathrm{~s}, 3 \mathrm{H}), 3.61(\mathrm{~s}, 3 \mathrm{H}), 3.45(\mathrm{~s}, 3 \mathrm{H}), 3.24(\mathrm{~s}, 3 \mathrm{H}), 2.53(\mathrm{~s}, 3 \mathrm{H}) ;{ }^{13} \mathrm{C} \mathrm{NMR}$ $\left(\mathrm{CDCl}_{3} 100 \mathrm{MHz}\right) \delta 165.6,158.3,134.9,123.5,115.5,56.0,48.0,46.7,45.9,42.1 ;{ }^{19} \mathrm{~F} \mathrm{NMR}\left(\mathrm{CDCl}_{3}\right.$ $376 \mathrm{MHz}) \delta-73.4(\mathrm{~d}, \mathrm{~J}=707 \mathrm{~Hz})$. Anal. Calcd. For $\mathrm{C}_{14} \mathrm{H}_{20} \mathrm{~F}_{6} \mathrm{~N}_{3} \mathrm{O}_{3} \mathrm{P}: \mathrm{C}, 39.72 ; \mathrm{H}, 4.76 ; \mathrm{N}, 9.93$. Found: C, 39.52; H, 4.46; N, 9.94.

4'-Chloro-5-dimethylamino-2-nitro-biphenyl-4-carboxylic acid methyl ester 21a

Yellow solid: mp $118-120^{\circ} \mathrm{C} ;{ }^{1} \mathrm{H}$ NMR $\left(\mathrm{CDCl}_{3}, 400 \mathrm{MHz}\right) \delta 8.50(\mathrm{~s}, 1 \mathrm{H}), 7.39(\mathrm{~m}, 2 \mathrm{H}), 7.24(\mathrm{~m}, 2 \mathrm{H})$, $6.65(\mathrm{~s}, 1 \mathrm{H}), 3.96(\mathrm{~s}, 3 \mathrm{H}), 3.03(\mathrm{~s}, 6 \mathrm{H}) ;{ }^{13} \mathrm{C} \mathrm{NMR}\left(\mathrm{CDCl}_{3} 100 \mathrm{MHz}\right) \delta 166.64,153.32,140.55,137.31$, 134.29, $130.72,129.14,128.65,118.03,116.30$, 52.62, 42.99. Anal. Calcd. For $\mathrm{C}_{16} \mathrm{H}_{15} \mathrm{ClN}_{2} \mathrm{O}_{4}: \mathrm{C}_{\text {, }}$ 57.41; H, 4.52; N, 8.37. Found: C, 57.23; H, 4.31; N, 8.19.

\section{5-Dimethylamino-2-nitro-biphenyl-4-carboxylic acid methyl ester 21b}

Yellow solid: $\mathrm{mp} 120-122^{\circ} \mathrm{C} ;{ }^{1} \mathrm{H} \mathrm{NMR}\left(\mathrm{CDCl}_{3}, 400 \mathrm{MHz}\right) \delta 8.49(\mathrm{~s}, 1 \mathrm{H}), 7.42(\mathrm{~m}, 2 \mathrm{H}), 7.30(\mathrm{~m}, 2 \mathrm{H})$, $6.71(\mathrm{~s}, 1 \mathrm{H}), 3.96(\mathrm{~s}, 3 \mathrm{H}), 3.03(\mathrm{~s}, 6 \mathrm{H}) ;{ }^{13} \mathrm{C} \mathrm{NMR}\left(\mathrm{CDCl}_{3}, 400 \mathrm{MHz}\right) \delta 166.94,153.55,141.71,138.80$, 137.54, 130.54, 128.43, 128.12, 127.75. 118.25. 116.11. 52.56. 43.02. Anal. Calcd. For $\mathrm{C}_{16} \mathrm{H}_{16} \mathrm{~N}_{2} \mathrm{O}_{4}$ : C, 63.99; H, 5.37; N, 9.33. Found: C, 63.71; H, 5.14; N, 9.12. 


\section{5-Dimethylamino-4'-methyl-2-nitro-biphenyl-4-carboxylic acid methyl ester 21c}

Yellow solid: mp $122-124^{\circ} \mathrm{C} ;{ }^{1} \mathrm{H}$ NMR $\left(\mathrm{CDCl}_{3}, 400 \mathrm{MHz}\right) \delta 8.47(\mathrm{~s}, 1 \mathrm{H}), 7.24(\mathrm{~d}, 2 \mathrm{H}, \mathrm{J}=8 \mathrm{~Hz}), 7.19$

$(\mathrm{d}, 2 \mathrm{H}, \mathrm{J}=8 \mathrm{~Hz}), 6.70(\mathrm{~s}, 1 \mathrm{H}), 3.95(\mathrm{~s}, 3 \mathrm{H}), 3.02(\mathrm{~s}, 6 \mathrm{H}), 2.42(\mathrm{~s}, 3 \mathrm{H}) ;{ }^{13} \mathrm{C} \mathrm{NMR}\left(\mathrm{CDCl}_{3} 100 \mathrm{MHz}\right) \delta$ 167.0, 153.6, 141.7, 138.1, 137.7, 135.8, 130.7, 129.2, 127.65, 118.3, 116.0, 52.24, 43.0, 21.3. Anal.

Calcd. For $\mathrm{C}_{17} \mathrm{H}_{18} \mathrm{~N}_{2} \mathrm{O}_{4}$ : C, 64.96; H, 5.77; N, 8.91. Found: C, 64.72; H, 5.70; N, 8.79.

\section{5-Dimethylamino-4'-methoxy-2-nitro-biphenyl-4-carboxylic acid methyl ester 21d}

Yellow solid: mp $133-135^{\circ} \mathrm{C} ;{ }^{1} \mathrm{H}$ NMR $\left(400 \mathrm{MHz} \mathrm{CDCl}_{3}\right) \delta 8.45(1 \mathrm{H}, \mathrm{s}), 7.24(2 \mathrm{H}, \mathrm{m}), 6.97(2 \mathrm{H}, \mathrm{m})$, $6.70(1 \mathrm{H}, \mathrm{s}), 3.94(3 \mathrm{H}, \mathrm{s}), 3.86(3 \mathrm{H}, \mathrm{s}), 3.02(6 \mathrm{H}, \mathrm{s}) .{ }^{13} \mathrm{C} \mathrm{NMR}\left(100 \mathrm{MHz} \mathrm{CDCl}_{3}\right)$ 166.9, 159.1, 153.5, 141.3, 137.7, 130.8, 130.5, 129.0, 118.2, 115.8, 113.9, 55.3, 52.5, 43.0. Anal. Calcd. For $\mathrm{C}_{17} \mathrm{H}_{18} \mathrm{~N}_{2} \mathrm{O}_{5}$ : C, 61.81; H, 5.49; N 8.48. Found: C, 61.77; H, 5.41; N, 8.28.

\section{4'-Chloro-5-hydroxy-2-nitro-biphenyl-4-carboxylic acid methyl ester 22a}

White solid: $\mathrm{mp} 114-115^{\circ} \mathrm{C} ;{ }^{1} \mathrm{H}$ NMR $\left(\mathrm{CDCl}_{3} 400 \mathrm{MHz}\right) \delta 11.25(\mathrm{~s}, 1 \mathrm{H}), 8.55(\mathrm{~s}, 1 \mathrm{H}), 7.41(\mathrm{~d}, 2 \mathrm{H}, \mathrm{J}=$ $8.4 \mathrm{~Hz}), 7.23(\mathrm{~d}, 2 \mathrm{H}, \mathrm{J}=8.4 \mathrm{~Hz}), 6.96(\mathrm{~s}, 1 \mathrm{H}), 4.04(\mathrm{~s}, 3 \mathrm{H}) ;{ }^{13} \mathrm{C} \mathrm{NMR}\left(\mathrm{CDCl}_{3} 100 \mathrm{MHz}\right) \delta 169.0,163.9$, 143.2, 140.6, 135.4, 135.0, 128.9, 127.8, 120.9, 111.4, 53.1. Anal. Calcd. For $\mathrm{C}_{14} \mathrm{H}_{10} \mathrm{ClNO}_{5}$ : C, 54.65; H, 3.28; N, 4.55. Found: C, 54.54; H, 3.00; N, 4.48 .

\section{5-Hydroxy-2-nitro-biphenyl-4-carboxylic acid methyl ester 22b}

Pale yellow solid: mp. 93-95 ${ }^{\circ} \mathrm{C} ;{ }^{1} \mathrm{H}$ NMR $\left(\mathrm{CDCl}_{3}, 400 \mathrm{MHz}\right) \delta 11.23(\mathrm{~s}, 1 \mathrm{H}), 8.54(\mathrm{~s}, 1 \mathrm{H}), 7.44(\mathrm{~m}$, 3H), $7.31(\mathrm{~m}, 2 \mathrm{H}), 7.01(\mathrm{~s}, 1 \mathrm{H}), 4.04(\mathrm{~s}, 3 \mathrm{H}) ;{ }^{13} \mathrm{C} \mathrm{NMR}\left(\mathrm{CDCl}_{3}, 100 \mathrm{MHz}\right) \delta 169.17,163.87,144.55$, 141.04, 136.94, 128.83, 128.72, 127.66, 127.54, 121.08, 111.21, 53.10. Anal. Calcd. For $\mathrm{C}_{14} \mathrm{H}_{11} \mathrm{NO}_{5}: \mathrm{C}_{\text {, }}$ 61.54; H, 4.06; N, 5.13. Found: C, 61.42; H, 3.76; N, 4.95.

5-Hydroxy-4'-methyl-2-nitro-biphenyl-4-carboxylic acid methyl ester 22c

Pale orange solid: mp. $121.123^{\circ} \mathrm{C} ;{ }^{1} \mathrm{H} \mathrm{NMR}\left(\mathrm{CDCl}_{3}, 400 \mathrm{MHz}\right) \delta 11.21(\mathrm{~s}, 1 \mathrm{H}), 8.51(\mathrm{~s}, 1 \mathrm{H}), 7.25(\mathrm{~d}$, $2 \mathrm{H}, \mathrm{J}=8.1 \mathrm{~Hz}), 7.20(\mathrm{~d}, 2 \mathrm{H}, \mathrm{J}=8.1 \mathrm{~Hz}), 7.00(\mathrm{~s}, 1 \mathrm{H}), 4.04(\mathrm{~s}, 3 \mathrm{H}), 2.42(\mathrm{~s}, 3 \mathrm{H}) ;{ }^{13} \mathrm{C} \mathrm{NMR}\left(\mathrm{CDCl}_{3}, 100\right.$ 
MHz) $\delta 169.1,163.7,144.5,414.1,138.9,133.8,129.4,127.5,127.4,120.9,110.9,53.0,21.3$; Anal.

Calcd. For $\mathrm{C}_{15} \mathrm{H}_{13} \mathrm{NO}_{5}$ : C, 62.72; H, 4.56; N, 4.88. Found: C, 62.65; H, 4.44; N, 4.89.

\section{5-Hydroxy-4'-methoxy-2-nitro-biphenyl-4-carboxylic acid methyl ester 22d}

Pale yellow solid: mp 136-138 ${ }^{\circ} \mathrm{C} ;{ }^{1} \mathrm{H}$ NMR $\left(\mathrm{CDCl}_{3}, 400 \mathrm{MHz}\right) \delta 11.19(\mathrm{~s}, 1 \mathrm{H}), 8.47(\mathrm{~s}, 1 \mathrm{H}), 7.24(\mathrm{~m}$, 2H), $6.98(\mathrm{~s}, 1 \mathrm{H}), 6.96(\mathrm{~m}, 2 \mathrm{H}), 4.03(\mathrm{~s}, 3 \mathrm{H}), 3.85(\mathrm{~s}, 3 \mathrm{H}) ;{ }^{13} \mathrm{C} \mathrm{NMR}\left(\mathrm{CDCl}_{3}, 100 \mathrm{MHz}\right) \delta 169.2,163.7$, 160.3, 144.1, 141.2, 129.0, 128.9, 127.6, 120.8, 114.3, 110.8, 55.4, 53.0. Anal. Calcd. For $\mathrm{C}_{15} \mathrm{H}_{13} \mathrm{NO6}_{\text {: }}$ C, 59.41; H, 4.32; N, 4.62. Found: C, 58.38; H, 4.03; N, 4.47.

\section{2-Nitro-1,3-bis(piperidino)trimethinium hexafluorophosphate 23b}

Light yellow solid: mp $162{ }^{\circ} \mathrm{C} ;{ }^{1} \mathrm{H}$ NMR $\left(500 \mathrm{MHz}, \mathrm{CD}_{3} \mathrm{CN}\right) \delta 8.40(2 \mathrm{H}, \mathrm{s}), 3.87(2 \mathrm{H}, \mathrm{t}, J=6 \mathrm{~Hz}), 3.54$ $(2 \mathrm{H}, \mathrm{t}, J=6 \mathrm{~Hz}), 1.80(12 \mathrm{H}, \mathrm{m}) ;{ }^{19} \mathrm{~F} \mathrm{NMR}\left(376 \mathrm{MHz}, \mathrm{CD}_{3} \mathrm{CN}\right)-723.1(\mathrm{~d}, J=703 \mathrm{~Hz})$. Anal. Calcd. For C, 39.30; H, 5.58, N, 10.58. Found: C, 39.64, H, 5.88, N, 10.50. HRMS Found: [M] 252.1695 $\mathrm{C}_{13} \mathrm{H}_{22} \mathrm{~N}_{3} \mathrm{O}_{2}$ requires 252.1712 .

2-Nitro-1,3-bis( $N$-diisopropyl)trimethinium hexafluorophosphate 23c

Light yellow solid. $v / \mathrm{cm}^{-1} 1603.87\left(v \mathrm{C}=\mathrm{N}^{+}\left({ }^{\mathrm{i}} \mathrm{Pr}\right)_{2}\right), 1465.48\left(v^{\mathrm{NO}} \mathrm{O}_{2}\right), 1278.53\left(\mathrm{v} \mathrm{NO}_{2}\right)^{1} \mathrm{H} \mathrm{NMR}(400$

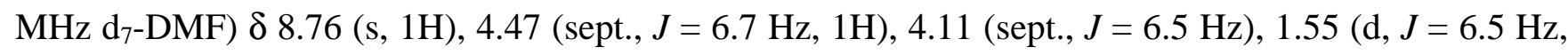
6H), $1.54(\mathrm{~d}, J=6.7 \mathrm{~Hz}, 6 \mathrm{H})$. Anal. Calcd. C, 41.96; H, 7.04, N, 9.79. Found: C, 42.00, H, 7.06, N, 9.86. HRMS [M] $]^{+}$284.2326. $\mathrm{C}_{15} \mathrm{H}_{30} \mathrm{~N}_{3} \mathrm{O}_{2}$ requires 284.2338.

Benzoic acid, 3-nitro-6-(1-piperidinyl)-, methyl ester $24 b^{3}$

Found: C, 59.05; H, 5.76; N, 10.13. $\mathrm{C}_{13} \mathrm{H}_{16} \mathrm{~N}_{2} \mathrm{O}_{4}$ requires, C, 59.08; H, 6.10; N, 10.60. ${ }^{1} \mathrm{H}$ NMR (400 $\left.\mathrm{MHz}_{\mathrm{CDCl}}\right) \delta 8.56(1 \mathrm{H}, \mathrm{d}, J=3 \mathrm{~Hz}), 8.16(1 \mathrm{H}, \mathrm{dd}, J=9,3 \mathrm{~Hz}), 6.95(1 \mathrm{H}, \mathrm{d}, J=9 \mathrm{~Hz}), 3.96(3 \mathrm{H}, \mathrm{s})$, $2.88(6 \mathrm{H}, \mathrm{s}), 2.39(3 \mathrm{H}, \mathrm{s}) .{ }^{13} \mathrm{C} \mathrm{NMR}\left(100 \mathrm{MHz}, \mathrm{CDCl}_{3}\right) \delta 167.0,156.2,138.2,128.8,127.6,119.5$, 117.1, 52.5, 52.4, 25.8, 23.8. HRMS $[\mathrm{M}+\mathrm{H}]^{+} 265.1178$.

Methan-d3-aminium,N-[3-[di(methyl-d3)amino]-2-nitro-2-propenylidene-1,3-d2]-N-(methyl-d3)-, hexafluorophosphate(1-) 31 
${ }^{2} \mathrm{H}$ NMR (77 MHz h 7 -DMF) $\delta 8.98$ (1D, s), 3.80 (3D, s), 3.40 (3D, s). ${ }^{13} \mathrm{C} \mathrm{NMR} \mathrm{(100} \mathrm{MHz} \mathrm{d} 7$-DMF) $\delta$ $159.4(\mathrm{t}, J=25 \mathrm{~Hz}), 116.0,47.6(\mathrm{t}, J=20 \mathrm{~Hz}), 42.6(\mathrm{t}, J=20 \mathrm{~Hz}) .{ }^{19} \mathrm{~F}$ NMR $\left(376 \mathrm{MHz} \mathrm{d}_{7}-\mathrm{DMF}\right) \delta-72.58$ $(\mathrm{d}, J=710 \mathrm{~Hz}) . v / \mathrm{cm}^{-1}$ 1599.3, 1488.2, 1298.9. HRMS [M] $]^{+}$186.1948; Anal Cald. For $\mathrm{C}_{7} \mathrm{D}_{14} \mathrm{~N}_{3} \mathrm{O}_{2}$ 186.1951. Found: $\mathrm{C}, 25.37 ; \mathrm{N}, 12.55 . \mathrm{C}_{7} \mathrm{D}_{14} \mathrm{~F}_{6} \mathrm{~N}_{3} \mathrm{O}_{2} \mathrm{P}$ requires, $\mathrm{C}, 25.38 ; \mathrm{N}, 12.69$.

\section{General procedure for the determination of isotope effects}

The isotope effects for the various additions were determined by reaction of a 1:1 mixture of $-\mathrm{H}$ and $-\mathrm{D}$ compounds with a deficiency (approximately 0.1 equiv) of methylacetoactetate in THF or acetontirile. ${ }^{4}$ The reactions were aged for $8 \mathrm{hr}$ at the desired temperature $\left(-10{ }^{\circ} \mathrm{C}\right.$ or $\left.25{ }^{\circ} \mathrm{C}\right)$. After the age the mixtures were diluted with acetonitrile to standard volume for quantitative HPLC analysis. The solutions were concentrated for ${ }^{1} \mathrm{H}$ and ${ }^{2} \mathrm{H} \mathrm{nmr}$ analysis at $500 \mathrm{MHz}$ and $77 \mathrm{MHz}$ respectively. A 20 second pulse delay was used for ${ }^{1} \mathrm{H}$ acquisition. The crude mixtures were then purified by flash chromatography and re-analyzed by ${ }^{1} \mathrm{H}$ NMR for verification of the crude reaction mixture data. The proton resonances at C-5 allow for determination of the mole fraction of each of the for reaction components. Reactions were performed in triplicate (nitro) or quadruplicate (dimethyliminium). The ${ }^{2} \mathrm{H}$ NMR shifts were referenced to the residual natural abundance deuterated solvent $\left(\delta 7.27\right.$ for $\mathrm{CDCl}_{3}$ and 1.94 for $\mathrm{CD}_{3} \mathrm{CN}$ ); deuterium shifts were not corrected for bulk magnetic susceptibility differences due to the protic solvent.

\section{Benzoic-2,6-d2 acid, 6-[di(methyl-d3)amino]-3-nitro-, methyl ester 32}

Found: $\mathrm{C}, 51.73 ; \mathrm{N}, 11.79 . \mathrm{C}_{10} \mathrm{H}_{4} \mathrm{D}_{8} \mathrm{~N}_{2} \mathrm{O}_{4}$ requires $\mathrm{C}, 51.71 ; \mathrm{N}, 11.19 .{ }^{1} \mathrm{H} \mathrm{NMR}\left(500 \mathrm{MHz} \mathrm{CDCl}_{3}\right) \delta$ $6.87(1 \mathrm{H}, \mathrm{s}), 3.96(3 \mathrm{H}, \mathrm{s}) .{ }^{2} \mathrm{H}$ NMR $\left(77 \mathrm{MHz}_{\mathrm{CHCl}}\right) \delta 8.55$ (s), 8.15 (s), 2.96 (s). HRMS [M+H] ${ }^{+}$ 233.1368. Required: 233.1365.

\section{Benzoic-2,4-d2 acid, 6-hydroxy-3-nitro-, methyl ester 34}


${ }^{1} \mathrm{H}$ NMR $\left(400 \mathrm{MHz} \mathrm{CDCl}_{3}\right) \delta 11.44(\mathrm{~s}, 1 \mathrm{H}), 7.10(1 \mathrm{H}, \mathrm{s}), 4.05(3 \mathrm{H}, \mathrm{s}) .{ }^{2} \mathrm{H} \mathrm{NMR}\left(77 \mathrm{MHz} \mathrm{CHCl}_{3}\right) \Lambda$ $8.67,8.22$.

Methanaminium,N-[3-[di(methyl)amino]-2-nitro-2-propenylidene-1,3-d2]-N-(methyl)-,hexafluorophosphate (1-) 35

colorless solid: mp 153-154 ${ }^{\circ} \mathrm{C}(\mathrm{EtOH})$; Anal. Calcd. For $\mathrm{C}_{7} \mathrm{H}_{12} \mathrm{D}_{2} \mathrm{~F}_{6} \mathrm{~N}_{2} \mathrm{P}$ : C, 26.34; H, 5.05; N, 13.16. Found: C, 26.40; H, 4.25; N, 13.00. ${ }^{1} \mathrm{H}$ NMR (500, $\left.\mathrm{MHz} \mathrm{CDCl}_{3}\right): \delta 6.84(\mathrm{~s}, 1 \mathrm{H}), 3.92(\mathrm{~s}, 3 \mathrm{H})$, $3.03(\mathrm{~s}, 3 \mathrm{H}) ;{ }^{2} \mathrm{H}$ NMR $\left(77 \mathrm{MHz}, \mathrm{CHCl}_{3}\right) \delta 8.57,8.17$.

Methanaminium,N,N'-[2-[(dimethylamino)-d1-methylene]-1,3-d2-propanediylidene],[N-methyl-, [tetrafluoroborate(1-)] [hexafluorophosphate(1-)] 37

${ }^{1} \mathrm{H}$ NMR (500 MHz CD $\left.3 \mathrm{CN}\right)$ residual protons. ${ }^{1} \mathrm{H}$ NMR $\left(500 \mathrm{MHz}, \mathrm{CD}_{3} \mathrm{CN}\right): \delta 3.52(\mathrm{~s}, 6 \mathrm{H}), 3.39$ (s, $6 \mathrm{H}) ;{ }^{2} \mathrm{H}$ NMR $\left(77 \mathrm{MHz}, \mathrm{CH}_{3} \mathrm{CN}\right) \delta 8.06 ;{ }^{13} \mathrm{C}$ NMR $\left(125 \mathrm{MHz}, \mathrm{CD}_{3} \mathrm{CN}\right) \Lambda 165.2(\mathrm{t}, \mathrm{J}=27.4 \mathrm{~Hz}), 91.7$, $50.2,4.3$.

Benzoic-2,6-d2-acid 2-dimethylamino-5-formyl-d-, methyl ester 38

${ }^{1} \mathrm{H}$ NMR $\left(500 \mathrm{MHz}, \mathrm{CDCl}_{3}\right) \delta 6.96(\mathrm{~s}, 1 \mathrm{H}), 3.93(\mathrm{~s}, 3 \mathrm{H}), 3.02(\mathrm{~s}, 6 \mathrm{H}) ;{ }^{2} \mathrm{H}$ NMR $\left(77 \mathrm{MHz}, \mathrm{CHCl}_{3}\right) \Lambda$ $9.83,8.20,7.83$

Benzoic-4,6-d2- acid 5-formyl-d-2-hydroxy-, methyl ester 39

${ }^{1} \mathrm{H}$ NMR $\left(500 \mathrm{MHz} \mathrm{CDCl}_{3}\right): \delta 11.36(\mathrm{~s}, 1 \mathrm{H}), 7.12(\mathrm{~s}, 1 \mathrm{H}), 4.02(\mathrm{~s}, 3 \mathrm{H}) ;{ }^{2} \mathrm{H} \mathrm{NMR}\left(77 \mathrm{MHz} \mathrm{CHCl}_{3}\right) \Lambda$ $9.94,8.45,7.86,8.07$. 
Supporting Information for X-ray analysis of 21d

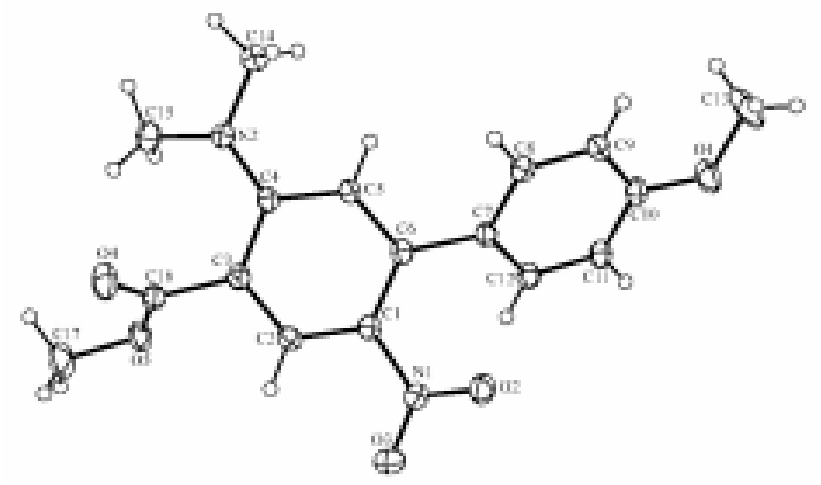

Figure 1. ORTEP representation of 21d. Non-hydrogen atoms are represented by ellipsoids corresponding to $20 \%$ probability. Hydrogen atoms have been drawn at an arbitrary size.

Table 1. Summary of Structure Determination of 21d

Table 2. Bond Distances in Angstroms

Table 3. Bond Angles in Degrees

Table 4. Fractional Atomic Coordinates and Equivalent Isotropic Displacement Parameters

Table 5. Anisotropic Displacement Parameters.

Table 1. Summary of Experimental Quantities of 21d

Formula

$\mathrm{Fw}$

Crystal colour

Crystal dimen. (mm)

Lattice symmetry

Space group

$a,(\AA)$

b

c

$\alpha$

$\beta$
$\mathrm{C}_{17} \mathrm{H}_{18} \mathrm{~N}_{2} \mathrm{O}_{5}$

330.343

yellowish

$0.10 \times 0.20 \times 0.33$

monoclinic

$P 2{ }_{1} / c$

9.152(2)

10.857(1)

16.9778(9)

90

104.200(8) 
$D_{\text {calc }}\left(\mathrm{Mg} \mathrm{m}^{-3}\right)$

1.342

Radiation $(K \alpha)$

$\mathrm{Cu}$

Wavelength $(\AA)$

1.54184

Temperature (K)

296

$\mu\left(\mathrm{mm}^{-1}\right)$

0.83

Diffractometer

Rigaku AFC5

Reflections measured

3322

Resolution $(\AA)$

0.82

Unique reflections

3322

$R_{\text {int }}$

0.027

Absorp. corr.

none

Reflections used

2950

Refl. obsd. criterion

$>2 \sigma(I)$

Variables

217

Refined on

$F^{2}$

$R$

0.060

$R_{w}$

0.180

S

1.04

residual peak $\left(\mathrm{e}^{-3}\right)$

$0.33(5)$

\section{Computer programs:}

Solution

SHELXS-97

Refinement

SHELXL-97 
Table 2. Bond Distances in $\AA$

\begin{tabular}{llll}
$\mathrm{O} 1-\mathrm{C} 10$ & $1.359(4)$ & $\mathrm{C} 2-\mathrm{C} 3$ & $1.381(4)$ \\
$\mathrm{O} 1-\mathrm{C} 13$ & $1.411(5)$ & $\mathrm{C} 3-\mathrm{C} 4$ & $1.426(4)$ \\
$\mathrm{O} 2-\mathrm{N} 1$ & $\mathrm{C} 3-\mathrm{C} 16$ & $1.495(4)$ \\
$\mathrm{O} 3-\mathrm{N} 1$ & $\mathrm{C} 4-\mathrm{C} 5$ & $1.412(4)$ \\
$\mathrm{O} 4-\mathrm{C} 16$ & $1.225(3)$ & $\mathrm{C} 5-\mathrm{C} 6$ & $1.378(4)$ \\
$\mathrm{O} 5-\mathrm{C} 16$ & $\mathrm{C} 6-\mathrm{C} 7$ & $1.487(4)$ \\
$\mathrm{O} 5-\mathrm{C} 17$ & $\mathrm{C} 7-\mathrm{C} 8$ & $1.391(4)$ \\
$\mathrm{N} 1-\mathrm{C} 1$ & $\mathrm{C} 7-\mathrm{C} 12$ & $1.395(4)$ \\
$\mathrm{N} 2-\mathrm{C} 4$ & $1.325(4)$ & $\mathrm{C} 8-\mathrm{C} 9$ & $1.388(4)$ \\
$\mathrm{N} 2-\mathrm{C} 15$ & $1.443(4)$ & $\mathrm{C} 9-\mathrm{C} 10$ & $1.373(4)$ \\
$\mathrm{N} 2-\mathrm{C} 14$ & $1.353(4)$ & $\mathrm{C} 10-\mathrm{C} 11$ & $1.393(5)$ \\
$\mathrm{C} 1-\mathrm{C} 2$ & $1.443(4)$ & $\mathrm{C} 11-\mathrm{C} 12$ & $1.376(4)$ \\
$\mathrm{C} 1-\mathrm{C} 6$ & $1.449(4)$ & & \\
\hline
\end{tabular}

Numbers in parentheses are estimated standard deviations in the least significant digits 
Table 3. Bond Angles in Degrees

\begin{tabular}{|c|c|c|c|}
\hline $\mathrm{C} 10-\mathrm{O} 1-\mathrm{C} 13$ & $118.5(3)$ & $\mathrm{C} 6-\mathrm{C} 5-\mathrm{C} 4$ & $124.8(3)$ \\
\hline $\mathrm{C} 16-\mathrm{O} 5-\mathrm{C} 17$ & $116.1(3)$ & $\mathrm{C} 5-\mathrm{C} 6-\mathrm{C} 1$ & $116.3(3)$ \\
\hline $\mathrm{O} 2-\mathrm{N} 1-\mathrm{O} 3$ & $121.6(3)$ & $\mathrm{C} 5-\mathrm{C} 6-\mathrm{C} 7$ & $117.8(3)$ \\
\hline $\mathrm{O} 2-\mathrm{N} 1-\mathrm{C} 1$ & 119.3(3) & $\mathrm{C} 1-\mathrm{C} 6-\mathrm{C} 7$ & $125.3(3)$ \\
\hline $\mathrm{O} 3-\mathrm{N} 1-\mathrm{C} 1$ & 119.1(3) & $\mathrm{C} 8-\mathrm{C} 7-\mathrm{C} 12$ & $117.5(3)$ \\
\hline $\mathrm{C} 4-\mathrm{N} 2-\mathrm{C} 15$ & $124.2(3)$ & $\mathrm{C} 8-\mathrm{C} 7-\mathrm{C} 6$ & $120.8(3)$ \\
\hline $\mathrm{C} 4-\mathrm{N} 2-\mathrm{C} 14$ & $120.5(3)$ & $\mathrm{C} 12-\mathrm{C} 7-\mathrm{C} 6$ & $121.4(3)$ \\
\hline $\mathrm{C} 15-\mathrm{N} 2-\mathrm{C} 14$ & $114.8(3)$ & $\mathrm{C} 9-\mathrm{C} 8-\mathrm{C} 7$ & $121.5(3)$ \\
\hline $\mathrm{C} 2-\mathrm{C} 1-\mathrm{C} 6$ & $121.0(3)$ & $\mathrm{C} 10-\mathrm{C} 9-\mathrm{C} 8$ & $120.1(3)$ \\
\hline $\mathrm{C} 2-\mathrm{C} 1-\mathrm{N} 1$ & $116.5(3)$ & $\mathrm{O} 1-\mathrm{C} 10-\mathrm{C} 9$ & $125.4(3)$ \\
\hline $\mathrm{C} 6-\mathrm{C} 1-\mathrm{N} 1$ & $122.3(3)$ & $\mathrm{O} 1-\mathrm{C} 10-\mathrm{C} 11$ & $115.5(3)$ \\
\hline $\mathrm{C} 3-\mathrm{C} 2-\mathrm{C} 1$ & $122.0(3)$ & $\mathrm{C} 9-\mathrm{C} 10-\mathrm{C} 11$ & $119.1(3)$ \\
\hline $\mathrm{C} 2-\mathrm{C} 3-\mathrm{C} 4$ & $119.1(3)$ & $\mathrm{C} 12-\mathrm{C} 11-\mathrm{C} 10$ & $120.6(3)$ \\
\hline $\mathrm{C} 2-\mathrm{C} 3-\mathrm{C} 16$ & $116.0(3)$ & $\mathrm{C} 11-\mathrm{C} 12-\mathrm{C} 7$ & $121.0(3)$ \\
\hline $\mathrm{C} 4-\mathrm{C} 3-\mathrm{C} 16$ & $124.4(3)$ & $\mathrm{O} 4-\mathrm{C} 16-\mathrm{O} 5$ & $124.4(3)$ \\
\hline $\mathrm{N} 2-\mathrm{C} 4-\mathrm{C} 5$ & $119.4(3)$ & $\mathrm{O} 4-\mathrm{C} 16-\mathrm{C} 3$ & $124.7(3)$ \\
\hline $\mathrm{N} 2-\mathrm{C} 4-\mathrm{C} 3$ & $124.0(3)$ & $\mathrm{O} 5-\mathrm{C} 16-\mathrm{C} 3$ & $110.8(3)$ \\
\hline $\mathrm{C} 5-\mathrm{C} 4-\mathrm{C} 3$ & $116.6(3)$ & & \\
\hline
\end{tabular}

Numbers in parentheses are estimated standard deviations in the least significant digits. 
Table 4. Fractional Atomic Coordinates and Equivalent Isotropic Displacement Parameters

$x$

$\mathrm{O} 1$

$\mathrm{O} 2$

$\mathrm{O} 3$

$\mathrm{O} 4$

O5

N1

N2

C1

C2

C3

C4

C5

C6

C7

C8

C9

C10

C11

C12

C13

C14

C15

C16

C17

H2

H5

H8
$0.9906(3)$

0.6657(3)

0.6513(3)

0.1254(3)

0.3352(3)

0.6277(3)

0.2553(3)

$0.5486(3)$

0.4591(3)

0.3606(3)

0.3504(3)

0.4501(3)

0.5501(3)

0.6631(3)

0.6207(4)

$0.7265(4)$

0.8772(4)

0.9217(3)

$0.8165(3)$

$0.9550(5)$

0.2287(5)

0.1903(4)

$0.2580(4)$

$0.2481(5)$

0.4656

0.4482

0.5190 $y$

0.0610(3)

0.4100(2)

$0.4622(3)$

$0.2139(3)$

$0.2016(3)$

0.3882(3)

$-0.0174(2)$

$0.2752(3)$

$0.2750(3)$

0.1800(3)

0.0789(3)

0.0803(3)

0.1739(3)

$0.1526(3)$

$0.1010(3)$

0.0689(3)

0.0877(3)

0.1399(3)

0.1720(3)

$-0.0018(6)$

$-0.1029(4)$

$-0.0534(4)$

0.1992(3)

$0.2222(5)$

0.3409

0.0131

0.0878 $z$

$0.68214(16)$

$0.83403(16) \quad 0.0602(7)$

$0.95374(17) \quad 0.0754(9)$

0.99524(16) $\quad 0.0696(8)$

1.09436(14) 0.0597(7)

$0.89697(17) \quad 0.0481(7)$

0.90431(16) 0.0454(7)

$0.90378(19) \quad 0.0408(7)$

0.95882(19) $\quad 0.0436(8)$

$0.96222(18) \quad 0.0411(7)$

$0.90744(17) \quad 0.0387(7)$

$0.85582(19) \quad 0.0415(7)$

$0.85240(18) \quad 0.0390(7)$

$0.80410(18) \quad 0.0397(7)$

$0.7268(2) \quad 0.0464(8)$

$0.6843(2) \quad 0.0486(8)$

$0.7182(2) \quad 0.0456(8)$

0.79532(19) $\quad 0.0463(8)$

$0.83728(19) \quad 0.0433(7)$

0.6071(3) 0.1024(19)

0.8367(3) $\quad 0.0691(11)$

0.9702(2) $\quad 0.0600(10)$

1.01740(19) 0.0440(7)

1.1541(3) $\quad 0.0860(15)$

$0.9945 \quad 0.052$

$0.8216 \quad 0.050$

$0.7031 \quad 0.056$ 


\begin{tabular}{lllll} 
H9 & 0.6953 & 0.0346 & 0.6327 & 0.058 \\
H11 & 1.0235 & 0.1531 & 0.8187 & 0.056 \\
H12 & 0.8482 & 0.2072 & 0.8886 & 0.052 \\
H13A & 1.0451 & -0.0140 & 0.5887 & 0.154 \\
H13B & 0.8845 & 0.0461 & 0.5678 & 0.154 \\
H13C & 0.9111 & -0.0803 & 0.6138 & 0.154 \\
H14A & 0.1585 & -0.1649 & 0.8441 & 0.104 \\
H14B & 0.3220 & -0.1412 & 0.8342 & 0.104 \\
H14C & 0.1880 & -0.0591 & 0.7870 & 0.104 \\
H15A & 0.1263 & -0.1237 & 0.9540 & 0.090 \\
H15B & 0.1320 & 0.0135 & 0.9833 & 0.090 \\
H15C & 0.2693 & -0.0741 & 1.0169 & 0.090 \\
H17A & 0.3144 & 0.2231 & 1.2075 & 0.129 \\
H17B & 0.1755 & 0.1574 & 1.1505 & 0.129 \\
H17C & 0.1968 & 0.2999 & 1.1436 & 0.129 \\
\hline
\end{tabular}

$U e q=(1 / 3) \Sigma_{i} \Sigma_{j} U_{i j} a_{i}^{*} a_{j}^{*} \mathbf{a}_{i} \cdot \mathbf{a}_{j}$ 
Table 5. Anisotropic Displacement Parameters

U11

O1

$\mathrm{O} 2$

$\mathrm{O} 3$

$\mathrm{O} 4$

O5

N1

$\mathrm{N} 2$

C1

$\mathrm{C} 2$

C3

C4

C5

C6

C7

C8

C9

C10

C11 0.0346(16)

$\begin{array}{lll}\mathrm{C} 12 & 0.0412(16) & 0.0498(19)\end{array}$

C13 0.069(3)

C14 0.077(3)

C15 0.070(2)

C16 0.0387(16)

C17
U22

$$
\text { 0.094(2) }
$$

$0.0528(14)$

$0.0651(18)$

$0.104(2)$

$0.0877(19)$

$0.0475(16)$

$0.0451(15)$

$0.0432(18)$

0.0462(19)

0.0491(19)

$0.0424(17)$

$0.0443(17)$

$0.0438(18)$

0.0393(16)

$0.050(2)$

$0.056(2)$

0.0506(19)

$0.057(2)$

$0.0498(19)$

$0.168(5)$

$0.062(2)$

$0.062(2)$

0.0508(19)

$0.130(4)$
U33

$0.0629(16)$

$0.0668(16)$

$0.0746(18)$

$0.0647(16)$

$0.0408(13)$

$0.0549(17)$

$0.0481(15)$

$0.0436(17)$

$0.0399(17)$

0.0383(16)

$0.0387(16)$

$0.0453(17)$

$0.0362(15)$

0.0410(16)

$0.0515(19)$

0.0439(17)

$0.0491(18)$

0.0491(19)

0.0393(16)

$0.077(3)$

$0.080(3)$

$0.055(2)$

0.0443(17)

$0.055(2)$
U23

$-0.0131(14)$

0.0094(13)

$-0.0263(15)$

$0.0009(15)$

$-0.0116(13)$

$-0.0023(14)$

$-0.0022(12)$

$-0.0006(14)$

$-0.0071(14)$

$-0.0001(14)$

0.0007(13)

$-0.0064(14)$

$0.0026(13)$

$0.0032(13)$

$-0.0048(16)$

$-0.0112(15)$

$0.0033(15)$

$0.0028(16)$

$0.0015(14)$

$-0.050(3)$

$-0.023(2)$

$0.0074(18)$

$-0.0028(15)$

$-0.026(3)$
U13

U12

0.0278(12) $\quad 0.0075(14)$

$0.0368(14) \quad-0.0006(13)$

$0.0331(16) \quad-0.0306(16)$

$0.0188(12) \quad 0.0088(14)$

$0.0202(11) \quad-0.0063(13)$

$0.0177(13) \quad-0.0048(13)$

$0.0223(12) \quad-0.0064(12)$

$0.0121(13) \quad-0.0004(14)$

$0.0132(14) \quad-0.0012(15)$

$0.0106(12) \quad-0.0002(14)$

$0.0106(12) \quad 0.0020(13)$

$0.0169(13) \quad 0.0007(14)$

$0.0106(12) \quad 0.0024(13)$

$0.0167(13) \quad 0.0013(14)$

$0.0154(14) \quad-0.0045(15)$

$0.0195(15) \quad-0.0040(16)$

0.0228(15) 0.0061(14)

$0.0139(14) \quad 0.0027(14)$

0.0105(13) 0.0006(14)

$0.031(2) \quad 0.023(3)$

$0.042(2) \quad-0.023(2)$

0.0286(18) -0.0112(19)

$0.0138(14) \quad-0.0020(14)$

$0.039(2) \quad-0.018(3)$ 


\section{Computational Studies}

Cartesian coordinates of reactants, transition structures, and products at B3LYP/6-31G* level for 1,5-Hydrogen migrations. Total energies (au) are given.

R49:1,3-Cyclohexadiene and TS50( transition structure)

R27: 1-Hydroxy-2-methylacetate-4-nitro-5-N-dimethylamine-1,3-cyclohexadiene and TS-51

R30: 1-N-dimethylamine-2-nitro-4-mehylacetate-5-hydroxy-1,3-cyclohexadiene.

\section{Cartesian coordinates of reactants, transition structures at B3LYP/6-31G* level for Electrocyclic ring closure. Total energies (au) are given.}

R52: $1,3,5-$ Hexatriene and TS

R26: Reactant with substituent $\mathrm{NO}_{2}$ ( for enol case) and TS

R28: Reactant with substituent $\mathrm{NO}_{2}$ ( for enamine case) and TS

R58: Reactant with substituent $\mathrm{C}=\mathrm{N}^{+} \mathrm{Me}_{2}$ ( for enol case) and TS-59

R55: Reactant with substituent $\mathrm{C}=\mathrm{N}^{+} \mathrm{Me}_{2}$ ( for enamine case) and TS-56 


\section{Cartesian coordinates of reactants, transition structures, and products at B3LYP/6-31G* level for 1,5-Hydrogen migrations. Total energies (au) are given}

R49: 1,3-Cyclohexadiene HF=-233.4189363

$\begin{array}{llll}\mathrm{C} & 0.000000 & 0.000000 & 0.000000 \\ \mathrm{H} & 0.000000 & 0.000000 & 1.087399 \\ \mathrm{C} & 1.158096 & 0.000000 & -0.686840 \\ \mathrm{H} & 2.111694 & 0.016364 & -0.165156 \\ \mathrm{C} & 1.157664 & -0.050663 & -2.153483 \\ \mathrm{H} & 2.090731 & -0.263928 & -2.668920 \\ \mathrm{C} & 0.029052 & 0.188001 & -2.847897 \\ \mathrm{H} & 0.036183 & 0.187267 & -3.935272 \\ \mathrm{C} & -1.248370 & 0.552874 & -2.125729 \\ \mathrm{H} & -1.288722 & 1.651115 & -2.025862 \\ \mathrm{H} & -2.125770 & 0.269244 & -2.717347 \\ \mathrm{C} & -1.320474 & -0.093730 & -0.730776 \\ \mathrm{H} & -1.586073 & -1.160038 & -0.831661 \\ \mathrm{H} & -2.124257 & 0.365362 & -0.144724\end{array}$

TS50 HF=-233.347295

$\begin{array}{llll}\text { C } & -1.123776 & -0.264197 & -0.643589 \\ \text { C } & -1.187906 & -0.024899 & 0.748044 \\ \text { C } & 0.000162 & 0.224484 & 1.440567 \\ \text { C } & 1.188010 & -0.024837 & 0.747799 \\ \text { C } & 1.123630 & -0.264416 & -0.643899 \\ \text { C } & -0.000139 & 0.453070 & -1.382861 \\ \text { H } & 0.000277 & 0.374150 & 2.516377 \\ \text { H } & -2.111192 & -0.202164 & 1.294787 \\ \text { H } & 0.000021 & -1.242861 & -0.628449 \\ \mathrm{H} & 2.111504 & -0.201708 & 1.294326\end{array}$




\begin{tabular}{|c|c|c|c|}
\hline $\mathrm{H}$ & -0.000022 & 1.549508 & -1.277504 \\
\hline$H$ & -0.000343 & 0.193097 & -2.444927 \\
\hline H & -2.036416 & -0.532636 & -1.175269 \\
\hline & 2.036278 & -0.532623 & -1.175706 \\
\hline
\end{tabular}

R27: 1-Hydroxy-2-methylacetate-4-nitro-5-N-dimethylamine-1,3-cyclohexadiene HF=-874.9954112

$\begin{array}{llll}\mathrm{C} & -0.561645 & -0.538174 & 1.680206 \\ \mathrm{C} & 0.850617 & -0.292375 & 1.210097 \\ \mathrm{C} & 1.185330 & -0.134307 & -0.084892 \\ \mathrm{C} & 0.211644 & -0.243557 & -1.156734 \\ \mathrm{C} & -1.048283 & -0.713755 & -0.853044 \\ \mathrm{C} & -1.366768 & -1.208093 & 0.527946 \\ \mathrm{H} & 2.208407 & 0.102293 & -0.354596 \\ \mathrm{H} & -2.444403 & -1.124399 & 0.698589 \\ \mathrm{H} & -1.136200 & -2.284113 & 0.528323 \\ \mathrm{O} & -2.055241 & -0.848469 & -1.712932 \\ \mathrm{H} & -1.742686 & -0.551116 & -2.598653 \\ \mathrm{~N} & -1.130978 & 0.684945 & 2.247747 \\ \mathrm{C} & -2.217765 & 0.446785 & 3.185351 \\ \mathrm{H} & -3.147803 & 0.070295 & 2.715904 \\ \mathrm{H} & -1.897826 & -0.274129 & 3.944468 \\ \mathrm{H} & -2.464298 & 1.384773 & 3.694431 \\ \mathrm{C} & -1.424854 & 1.752645 & 1.305103 \\ \mathrm{O} & -0.544315 & 1.970297 & 0.694648 \\ \mathrm{H} & -2.273730 & 1.532652 & 0.628463 \\ \mathrm{H} & -1.676030 & 2.661038 & 1.863320 \\ \mathrm{H} & 1.884290 & -0.183381 & 2.231390 \\ \mathrm{O} & 1.573140 & -0.495712 & 3.385012 \\ \mathrm{O} & 3.011342 & 0.189587 & 1.894438 \\ \mathrm{O} & 0.667216 & 0.141367 & -2.501558 \\ \mathrm{H} & 1.784369 & 0.510175 & -2.792331 \\ \mathrm{H} & -0.329044 & 0.041205 & -3.450613\end{array}$




\begin{tabular}{|c|c|c|c|}
\hline $\mathrm{C}$ & 0.052731 & 0.396238 & -4.794576 \\
\hline 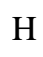 & 0.393114 & 1.433291 & -4.827895 \\
\hline & 0.854001 & -0.258353 & -5.144036 \\
\hline & -0.845579 & 0.264140 & -5.397763 \\
\hline & -0.521699 & -1.252551 & 2.504847 \\
\hline
\end{tabular}

\section{TS51 HF=-874.908195}

\begin{tabular}{|c|c|c|c|}
\hline $\mathrm{C}$ & -0.606875 & -0.143000 & -1.373777 \\
\hline $\mathrm{C}$ & 0.787284 & -0.252939 & -0.998814 \\
\hline $\mathrm{C}$ & 1.120068 & -0.427138 & 0.338913 \\
\hline $\mathrm{C}$ & 0.128100 & -0.286163 & 1.316035 \\
\hline $\mathrm{C}$ & -1.219468 & -0.168172 & 0.841041 \\
\hline $\mathrm{C}$ & -1.503250 & -0.941639 & -0.425665 \\
\hline $\mathrm{H}$ & 2.161366 & -0.454335 & 0.635270 \\
\hline $\mathrm{H}$ & -0.950047 & 0.915914 & -0.303570 \\
\hline $\mathrm{H}$ & -1.270105 & -2.017079 & -0.371824 \\
\hline $\mathrm{H}$ & -2.552844 & -0.814877 & -0.691019 \\
\hline $\mathrm{O}$ & -2.284217 & 0.026046 & 1.645317 \\
\hline $\mathrm{H}$ & -1.954341 & 0.333887 & 2.515622 \\
\hline $\mathrm{N}$ & -1.069029 & 0.019779 & -2.667246 \\
\hline $\mathrm{C}$ & -2.446384 & 0.448267 & -2.871867 \\
\hline $\mathrm{H}$ & -3.132180 & -0.388732 & -3.070837 \\
\hline $\mathrm{H}$ & -2.807124 & 1.003131 & -2.001120 \\
\hline $\mathrm{H}$ & -2.483113 & 1.127839 & -3.730403 \\
\hline $\mathrm{C}$ & -0.288063 & -0.155918 & -3.884128 \\
\hline $\mathrm{H}$ & 0.537272 & -0.850162 & -3.716097 \\
\hline $\mathrm{H}$ & -0.944760 & -0.606167 & -4.638294 \\
\hline $\mathrm{H}$ & 0.118955 & 0.781911 & -4.278422 \\
\hline $\mathrm{N}$ & 1.891862 & 0.195283 & -1.837211 \\
\hline $\mathrm{O}$ & 1.708677 & 1.187067 & -2.549805 \\
\hline $\mathrm{O}$ & 2.962622 & -0.408833 & -1.744710 \\
\hline $\mathrm{C}$ & 0.565690 & -0.102256 & 2.709376 \\
\hline
\end{tabular}




$\begin{array}{llll}\mathrm{O} & 1.682180 & -0.306135 & 3.135903 \\ \mathrm{O} & -0.451469 & 0.338666 & 3.525105 \\ \mathrm{C} & -0.096944 & 0.532193 & 4.907484 \\ \mathrm{H} & 0.237172 & -0.409670 & 5.348578 \\ \mathrm{H} & 0.701762 & 1.272322 & 4.991853 \\ \mathrm{H} & -1.005136 & 0.886691 & 5.395398\end{array}$

R30: 1-N-dimethylamine-2-nitro-4-mehylacetate-5-hydroxy-1,3-cyclohexadiene HF=-874.9877896

\begin{tabular}{llll}
$\mathrm{C}$ & -0.540861 & -0.270401 & 1.436732 \\
$\mathrm{C}$ & 0.781230 & -0.128430 & 0.996711 \\
$\mathrm{C}$ & 1.087464 & -0.143925 & -0.407043 \\
$\mathrm{C}$ & 0.142159 & -0.242660 & -1.373299 \\
$\mathrm{C}$ & -1.317369 & -0.360545 & -1.003909 \\
$\mathrm{C}$ & -1.498784 & -0.881713 & 0.429185 \\
$\mathrm{H}$ & 2.129079 & -0.030913 & -0.685878 \\
$\mathrm{H}$ & -2.539305 & -0.698926 & 0.700304 \\
$\mathrm{H}$ & -1.333016 & -1.967934 & 0.419242 \\
$\mathrm{O}$ & -1.998617 & 0.896020 & -1.086083 \\
$\mathrm{H}$ & -1.884935 & 1.195300 & -2.003937 \\
$\mathrm{~N}$ & -1.024614 & 0.119655 & 2.629325 \\
$\mathrm{C}$ & -2.300308 & -0.369320 & 3.157391 \\
$\mathrm{H}$ & -3.120921 & 0.325588 & 2.937119 \\
$\mathrm{H}$ & -2.541150 & -1.353976 & 2.758300 \\
$\mathrm{H}$ & -2.206303 & -0.463555 & 4.243051 \\
$\mathrm{C}$ & -0.382384 & 1.130967 & 3.465948 \\
$\mathrm{H}$ & 0.265090 & 1.759025 & 2.852112 \\
$\mathrm{H}$ & -1.172915 & 1.757249 & 3.893682 \\
$\mathrm{H}$ & 0.215236 & 0.683000 & 4.263651 \\
$\mathrm{~N}$ & 1.906082 & -0.075302 & 1.896570 \\
$\mathrm{O}$ & 1.752064 & -0.474346 & 3.064241 \\
$\mathrm{O}$ & 2.997279 & 0.300299 & 1.449312 \\
$\mathrm{H}$ & 0.543794 & -0.102700 & -2.780813 \\
$\mathrm{H}$ & 1.675374 & -0.114294 & -3.223658 \\
\hline
\end{tabular}




\begin{tabular}{|c|c|c|c|}
\hline Q & -0.555388 & 0.081439 & -3.583971 \\
\hline$C$ & -0.267484 & 0.239064 & -4.981380 \\
\hline & 0.361205 & 1.117819 & -5.148834 \\
\hline & 0.250031 & -0.642681 & -5.368167 \\
\hline & -1.235295 & 0.360230 & -5.469923 \\
\hline & -1.807515 & -1.085667 & -1.667855 \\
\hline
\end{tabular}




\section{Cartesian coordinates of reactants, transition structures at B3LYP/6-31G* level for Electrocyclic ring closure. Total energies (au) are given.}

\section{ELECTROCYCLIC RING CLOSURE}

52 (1,3,5-hexatriene) $\quad \mathrm{HF}=-233.3955067$

$\begin{array}{llll}\mathrm{C} & 2.894521 & -0.318884 & 0.000066 \\ \mathrm{H} & 3.405963 & 0.641335 & 0.000179 \\ \mathrm{H} & 3.521831 & -1.205045 & 0.000070 \\ \mathrm{C} & 1.553914 & -0.400823 & -0.000062 \\ \mathrm{H} & 1.092276 & -1.386558 & -0.000136 \\ \mathrm{C} & 0.677687 & 0.757542 & -0.000064 \\ \mathrm{H} & 1.182403 & 1.723262 & 0.000044 \\ \mathrm{C} & -0.677687 & 0.757542 & -0.000037 \\ \mathrm{H} & -1.182403 & 1.723262 & 0.000084 \\ \mathrm{C} & -1.553914 & -0.400823 & 0.000002 \\ \mathrm{H} & -1.092276 & -1.386558 & -0.000050 \\ \mathrm{H} & -2.894521 & -0.318884 & 0.000046 \\ \mathrm{H} & -3.405963 & 0.641335 & 0.000081 \\ \mathrm{H} & -3.521831 & -1.205045 & 0.000024\end{array}$

TS HF=-233.3472995

$\begin{array}{llll}\mathrm{C} & -0.106083 & -1.203716 & 1.140653 \\ \mathrm{C} & 0.188734 & 0.119843 & 1.482489 \\ \mathrm{C} & -0.106083 & 1.242319 & 0.702964 \\ \mathrm{C} & -0.106083 & 1.242319 & -0.702964 \\ \mathrm{C} & 0.188734 & 0.119843 & -1.482489 \\ \mathrm{C} & -0.106083 & -1.203716 & -1.140653 \\ \mathrm{H} & 0.841280 & 0.296276 & 2.340488 \\ \mathrm{H} & -0.016579 & 2.220000 & 1.175106\end{array}$




\begin{tabular}{|c|c|c|c|}
\hline $\mathrm{H}$ & -0.016579 & 2.220000 & -1.175106 \\
\hline $\mathrm{H}$ & 0.841280 & 0.296276 & -2.340488 \\
\hline $\mathrm{H}$ & 0.447050 & -1.999204 & -1.641147 \\
\hline $\mathrm{H}$ & -1.131155 & -1.467749 & -0.936255 \\
\hline $\mathrm{H}$ & 0.447050 & -1.999204 & 1.641147 \\
\hline $\mathrm{H}$ & -1.131155 & -1.467749 & 0.936255 \\
\hline
\end{tabular}

$\mathbf{R}=\mathrm{NO}_{2}$

26 (Enol_reactant) $\quad H F=-874.9653824$

\begin{tabular}{|c|c|c|c|}
\hline $\mathrm{N}$ & 2.221438 & -1.188370 & -0.779429 \\
\hline $\mathrm{N}$ & 1.726069 & 2.302212 & 0.235919 \\
\hline $\mathrm{C}$ & 1.248522 & 0.962278 & -0.068706 \\
\hline $\mathrm{C}$ & -0.198298 & 0.849389 & -0.130213 \\
\hline $\mathrm{C}$ & -1.005734 & -0.162004 & 0.294656 \\
\hline $\mathrm{C}$ & -0.597254 & -1.352599 & 1.101964 \\
\hline $\mathrm{C}$ & 0.268794 & -1.292628 & 2.122587 \\
\hline $\mathrm{C}$ & -2.453718 & -0.090734 & -0.043544 \\
\hline $\mathrm{C}$ & -4.263987 & 1.205517 & -0.816671 \\
\hline $\mathrm{C}$ & 2.257110 & 0.030587 & -0.228340 \\
\hline $\mathrm{C}$ & 3.346758 & -2.100295 & -0.596163 \\
\hline $\mathrm{C}$ & 1.122083 & -1.684644 & -1.602832 \\
\hline $\mathrm{O}$ & -3.216839 & -1.052194 & 0.028639 \\
\hline $\mathrm{O}$ & -2.874016 & 1.114578 & -0.458427 \\
\hline $\mathrm{O}$ & 2.946184 & 2.519310 & 0.285251 \\
\hline $\mathrm{O}$ & 0.872458 & 3.175886 & 0.437292 \\
\hline $\mathrm{H}$ & -0.694484 & 1.723657 & -0.536670 \\
\hline $\mathrm{H}$ & 0.493503 & -2.186998 & 2.693749 \\
\hline $\mathrm{H}$ & -4.496417 & 0.513845 & -1.630383 \\
\hline $\mathrm{H}$ & -4.895641 & 0.971420 & 0.043670 \\
\hline $\mathrm{H}$ & -4.411340 & 2.238101 & -1.132672 \\
\hline $\mathrm{H}$ & 3.239683 & 0.351041 & 0.104280 \\
\hline
\end{tabular}




\begin{tabular}{|c|c|c|c|}
\hline $\mathrm{H}$ & 4.159992 & -1.585298 & -0.081216 \\
\hline $\mathrm{H}$ & 3.043377 & -2.964534 & 0.006392 \\
\hline $\mathrm{H}$ & 0.402488 & -2.274959 & -1.026814 \\
\hline $\mathrm{H}$ & 0.607487 & -0.840792 & -2.063735 \\
\hline $\mathrm{H}$ & 1.549066 & -2.312583 & -2.391125 \\
\hline $\mathrm{H}$ & 0.749942 & -0.364077 & 2.401301 \\
\hline $\mathrm{H}$ & 3.705204 & -2.458771 & -1.567895 \\
\hline $\mathrm{O}$ & -1.150621 & -2.561274 & 0.755387 \\
\hline & -2.048397 & -2.366587 & 0.404122 \\
\hline
\end{tabular}

TS $\quad H F=-874.9447981$

\begin{tabular}{|c|c|c|c|}
\hline $\mathrm{C}$ & -1.846322 & -0.528627 & -0.571363 \\
\hline $\mathrm{C}$ & -1.104248 & 0.722388 & -0.449558 \\
\hline $\mathrm{C}$ & 0.274057 & 0.935046 & -0.475360 \\
\hline $\mathrm{C}$ & 1.319320 & 0.012673 & -0.403767 \\
\hline $\mathrm{C}$ & 1.062557 & -1.402233 & -0.552997 \\
\hline $\mathrm{C}$ & 0.065701 & -1.838949 & -1.392599 \\
\hline $\mathrm{H}$ & 0.562431 & 1.978621 & -0.393020 \\
\hline $\mathrm{H}$ & -0.169072 & -1.234236 & -2.257479 \\
\hline $\mathrm{H}$ & -2.072053 & -0.937399 & -1.545593 \\
\hline $\mathrm{N}$ & -2.610123 & -1.014965 & 0.402281 \\
\hline $\mathrm{C}$ & -2.550778 & -0.525903 & 1.773698 \\
\hline $\mathrm{H}$ & -3.345433 & 0.209005 & 1.943035 \\
\hline $\mathrm{H}$ & -2.668103 & -1.369469 & 2.460769 \\
\hline $\mathrm{H}$ & -1.586162 & -0.045637 & 1.944110 \\
\hline $\mathrm{C}$ & -3.676148 & -1.965206 & 0.109168 \\
\hline $\mathrm{H}$ & -3.653183 & -2.785547 & 0.833630 \\
\hline $\mathrm{H}$ & -4.651322 & -1.466336 & 0.166847 \\
\hline $\mathrm{H}$ & -3.543267 & -2.371870 & -0.895448 \\
\hline $\mathrm{H}$ & -0.168691 & -2.898147 & -1.439729 \\
\hline $\mathrm{O}$ & 1.732573 & -2.326854 & 0.173368 \\
\hline $\mathrm{H}$ & 2.452118 & -1.867846 & 0.649199 \\
\hline
\end{tabular}




\begin{tabular}{|c|c|c|c|}
\hline $\mathrm{N}$ & -1.931864 & 1.879637 & -0.171781 \\
\hline $\mathrm{O}$ & -1.409518 & 2.996937 & -0.101611 \\
\hline $\mathrm{O}$ & -3.161800 & 1.702251 & -0.055592 \\
\hline $\mathrm{C}$ & 2.633225 & 0.575046 & -0.052976 \\
\hline $\mathrm{O}$ & 3.526650 & -0.396562 & 0.352241 \\
\hline $\mathrm{O}$ & 2.949233 & 1.747647 & -0.075582 \\
\hline $\mathrm{C}$ & 4.833677 & 0.080816 & 0.711570 \\
\hline $\mathrm{H}$ & 5.405102 & -0.807153 & 0.984547 \\
\hline $\mathrm{H}$ & 5.297434 & 0.591162 & -0.135655 \\
\hline $\mathrm{H}$ & 4.770755 & 0.774490 & 1.553813 \\
\hline
\end{tabular}

28 (Enamin_reactant) HF=-933.710301

\begin{tabular}{llll}
$\mathrm{N}$ & -2.268193 & -2.017178 & -0.211913 \\
$\mathrm{C}$ & -1.354918 & -0.898536 & -0.134875 \\
$\mathrm{C}$ & 0.043177 & -1.253531 & -0.119734 \\
$\mathrm{C}$ & 1.138728 & -0.485597 & 0.128215 \\
$\mathrm{C}$ & 1.115596 & 0.944446 & 0.581404 \\
$\mathrm{C}$ & 1.007177 & 1.208726 & 1.898104 \\
$\mathrm{C}$ & 2.447010 & -1.186408 & 0.020108 \\
$\mathrm{C}$ & -1.802583 & 0.415091 & -0.222033 \\
$\mathrm{O}$ & 3.490016 & -0.352438 & 0.276823 \\
$\mathrm{O}$ & 2.605923 & -2.358655 & -0.266997 \\
$\mathrm{O}$ & -3.399134 & -1.823856 & -0.692771 \\
$\mathrm{O}$ & -1.864585 & -3.131703 & 0.140319 \\
$\mathrm{H}$ & 0.244992 & -2.301477 & -0.320618 \\
$\mathrm{H}$ & 1.008861 & 2.210450 & 2.311515 \\
$\mathrm{H}$ & 0.922484 & 0.388833 & 2.601262 \\
$\mathrm{~N}$ & 1.118686 & 1.934363 & -0.425974 \\
$\mathrm{C}$ & 1.178085 & 3.316568 & 0.024241 \\
$\mathrm{H}$ & 0.341386 & 3.527825 & 0.698145 \\
$\mathrm{H}$ & 2.113998 & 3.551404 & 0.558304 \\
$\mathrm{H}$ & 1.097373 & 3.978391 & -0.842896 \\
& & & \\
\hline & & &
\end{tabular}




$\begin{array}{llll}\mathrm{C} & 1.897884 & 1.684611 & -1.638925 \\ \mathrm{H} & 1.703375 & 0.674636 & -2.004138 \\ \mathrm{H} & 1.586120 & 2.394110 & -2.412264 \\ \mathrm{H} & 2.981134 & 1.791930 & -1.477286 \\ \mathrm{C} & 4.784975 & -0.963714 & 0.205563 \\ \mathrm{H} & 5.496274 & -0.170628 & 0.440288 \\ \mathrm{H} & 4.866763 & -1.778833 & 0.929647 \\ \mathrm{H} & 4.970800 & -1.364061 & -0.795187 \\ \mathrm{H} & -1.053403 & 1.136011 & -0.543834 \\ \mathrm{~N} & -2.984645 & 0.967280 & 0.075137 \\ \mathrm{C} & -4.038452 & 0.313812 & 0.843769 \\ \mathrm{H} & -3.596506 & -0.423187 & 1.516186 \\ \mathrm{H} & -4.542403 & 1.081157 & 1.440358 \\ \mathrm{H} & -4.761203 & -0.194173 & 0.200598 \\ \mathrm{C} & -3.264330 & 2.334222 & -0.355289 \\ \mathrm{H} & -3.588468 & 2.940516 & 0.498032 \\ \mathrm{H} & -2.365503 & 2.776224 & -0.789492 \\ \mathrm{H} & -4.058857 & 2.344697 & -1.111657\end{array}$

TS HF=-933.6931265

$\begin{array}{llll}\mathrm{C} & 1.965905 & 0.302853 & -0.753389 \\ \mathrm{C} & 1.386145 & -0.956614 & -0.304589 \\ \mathrm{C} & 0.032694 & -1.320745 & -0.273457 \\ \mathrm{C} & -1.089552 & -0.506045 & -0.312465 \\ \mathrm{C} & -0.937372 & 0.935562 & -0.495570 \\ \mathrm{C} & -0.080202 & 1.361487 & -1.499735 \\ \mathrm{H} & -0.153158 & -2.374113 & -0.087630 \\ \mathrm{H} & 0.018254 & 0.732933 & -2.375547 \\ \mathrm{H} & 2.056186 & 0.505805 & -1.812119 \\ \mathrm{~N} & 2.784227 & 1.038874 & 0.001084 \\ \mathrm{C} & 2.921438 & 0.842802 & 1.437047\end{array}$




\begin{tabular}{|c|c|c|c|}
\hline $\mathrm{H}$ & 3.796945 & 0.220097 & 1.650750 \\
\hline $\mathrm{H}$ & 3.025712 & 1.817252 & 1.925633 \\
\hline $\mathrm{H}$ & 2.032885 & 0.334956 & 1.816006 \\
\hline $\mathrm{C}$ & 3.724893 & 1.967763 & -0.609440 \\
\hline $\mathrm{H}$ & 3.437881 & 2.157035 & -1.646233 \\
\hline $\mathrm{H}$ & 3.726892 & 2.915307 & -0.060115 \\
\hline $\mathrm{H}$ & 4.738191 & 1.547141 & -0.592518 \\
\hline $\mathrm{H}$ & 0.176957 & 2.408566 & -1.630036 \\
\hline$N$ & -1.538610 & 1.810279 & 0.369436 \\
\hline $\mathrm{N}$ & 2.339971 & -1.949040 & 0.115145 \\
\hline $\mathrm{O}$ & 1.939059 & -3.063723 & 0.475648 \\
\hline $\mathrm{O}$ & 3.555638 & -1.656545 & 0.068498 \\
\hline $\mathrm{C}$ & -2.393214 & -1.176238 & -0.160794 \\
\hline $\mathrm{O}$ & -3.446526 & -0.311800 & -0.270693 \\
\hline $\mathrm{O}$ & -2.560383 & -2.372322 & 0.007159 \\
\hline $\mathrm{C}$ & -4.737101 & -0.927014 & -0.180553 \\
\hline $\mathrm{H}$ & -5.457379 & -0.114154 & -0.288428 \\
\hline $\mathrm{H}$ & -4.872132 & -1.664597 & -0.976605 \\
\hline $\mathrm{H}$ & -4.866058 & -1.427980 & 0.783309 \\
\hline $\mathrm{C}$ & -1.992569 & 1.433609 & 1.699210 \\
\hline $\mathrm{H}$ & -1.583443 & 2.132107 & 2.442201 \\
\hline $\mathrm{H}$ & -3.087198 & 1.451570 & 1.767740 \\
\hline $\mathrm{H}$ & -1.644586 & 0.429312 & 1.941773 \\
\hline $\mathrm{C}$ & -1.667843 & 3.221749 & 0.052009 \\
\hline $\mathrm{H}$ & -2.577949 & 3.613280 & 0.519829 \\
\hline $\mathrm{H}$ & -0.816676 & 3.817831 & 0.416655 \\
\hline $\mathrm{H}$ & -1.752059 & 3.356948 & -1.028855 \\
\hline
\end{tabular}


$\mathrm{R}=\mathrm{C}=\mathrm{N}^{+} \mathrm{Me}_{2}$

58 (Enol_reactant) $\quad H F=-842.9340047$

C

$\begin{array}{llll}\text { C } & -0.268826 & 0.663941 & -0.322590\end{array}$

$\begin{array}{llll}\text { C } & -1.403555 & 0.008618 & 0.003343\end{array}$

$\begin{array}{llll}\text { C } & -1.462977 & -1.345550 & 0.618349\end{array}$

$\begin{array}{llll}\text { C } & -1.196037 & -1.594838 & 1.905592\end{array}$

$\begin{array}{llll}\text { C } & -2.688021 & 0.730237 & -0.300568\end{array}$

$\begin{array}{llll}\text { C } & 1.296909 & -1.219344 & -0.514665\end{array}$

$\begin{array}{llll}\text { O } & -3.758651 & 0.009414 & 0.056949\end{array}$

$\begin{array}{lllll}\text { O } & -2.738141 & 1.840777 & -0.792459\end{array}$

$\mathrm{H} \quad-0.416908 \quad 1.658856-0.733065$

$\mathrm{H} \quad-1.261654 \quad-2.596832 \quad 2.322701$

$\mathrm{H} \quad \begin{array}{llll}-0.958826 & -0.787170 & 2.587154\end{array}$

$\begin{array}{llll}\text { C } & -5.038766 & 0.626084 & -0.195965\end{array}$

H $\quad \begin{array}{llll}-5.779149 & -0.097752 & 0.142262\end{array}$

$\begin{array}{llll}\mathrm{H} & -5.124963 & 1.560466 & 0.363443\end{array}$

$\mathrm{H} \quad \begin{array}{llll}\mathrm{H} & -5.154182 & 0.831332 & -1.262641\end{array}$

$\begin{array}{lllll}\mathrm{H} & 0.456497 & -1.736541 & -0.970088\end{array}$

N $\quad \begin{array}{llll}2.335969 & -2.007635 & -0.277999\end{array}$

$\begin{array}{llll}\text { C } & 3.500590 & -1.657150 & 0.540200\end{array}$

$\mathrm{H} \quad 3.243256-0.845961 \quad 1.223292$

$\begin{array}{llll}\mathrm{H} & 3.787521 & -2.536545 & 1.123113\end{array}$

$\mathrm{H} \quad 4.354926 \quad-1.365367 \quad-0.080057$

C $\quad 2.341606 \quad-3.383983 \quad-0.796326$

$\begin{array}{llll}\mathrm{H} & 2.437841 & -4.088426 & 0.035867\end{array}$

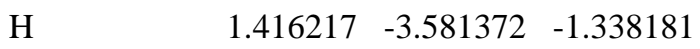

H $\quad 3.189293 \quad-3.520622 \quad-1.475566$

$\begin{array}{llll}\text { C } & 2.216966 & 1.033330 & -0.231615\end{array}$

$\begin{array}{llll}\mathrm{H} & 3.170175 & 0.642512 & -0.572721\end{array}$ 


$\begin{array}{llll}\mathrm{N} & 2.276138 & 2.320493 & 0.096458 \\ \mathrm{C} & 1.214234 & 3.074627 & 0.776263 \\ \mathrm{H} & 1.684466 & 3.718230 & 1.525208 \\ \mathrm{H} & 0.663608 & 3.704231 & 0.070077 \\ \mathrm{H} & 0.523958 & 2.395262 & 1.273283 \\ \mathrm{C} & 3.482043 & 3.104154 & -0.199358 \\ \mathrm{H} & 4.234408 & 2.474837 & -0.677030 \\ \mathrm{H} & 3.230223 & 3.927158 & -0.876577 \\ \mathrm{H} & 3.889309 & 3.522893 & 0.726503 \\ \mathrm{O} & -1.806188 & -2.298155 & -0.311382 \\ \mathrm{H} & -2.070795 & -3.113262 & 0.148467\end{array}$

TS-59 HF=-842.9161208

$\begin{array}{lrrr}\mathrm{C} & -1.408101 & -1.248956 & -.692260 \\ \mathrm{C} & -1.082378 & .182533 & -.444495 \\ \mathrm{C} & .256240 & .684130 & -.426702 \\ \mathrm{C} & 1.426515 & -.009943 & -.253193 \\ \mathrm{C} & 1.411336 & -1.456930 & -.208455 \\ \mathrm{C} & .671747 & -2.167777 & -1.129039 \\ \mathrm{H} & .393522 & 1.756751 & -.454649 \\ \mathrm{H} & .572862 & -1.754434 & -2.124241 \\ \mathrm{H} & -1.517531 & -1.577650 & -1.718979 \\ \mathrm{~N} & -2.150151 & -1.946697 & .170142 \\ \mathrm{C} & -2.107549 & -1.728702 & 1.620448 \\ \mathrm{H} & -3.119200 & -1.572835 & 2.007540 \\ \mathrm{H} & -1.683081 & -2.615665 & 2.105377 \\ \mathrm{H} & -1.491979 & -.860763 & 1.850571 \\ \mathrm{C} & -2.782516 & -3.203435 & -.240055 \\ \mathrm{H} & -2.839194 & -3.258202 & -1.328291 \\ \mathrm{H} & -2.210216 & -4.058962 & .138836 \\ \mathrm{H} & -3.794968 & -3.253340 & .171209\end{array}$




$\begin{array}{llll}\mathrm{H} & .577478 & -3.249576 & -1.046847 \\ \mathrm{C} & 2.658676 & .808094 & -.040805 \\ \mathrm{O} & 3.769478 & .058204 & -.013822 \\ \mathrm{O} & 2.645179 & 2.020800 & .058859 \\ \mathrm{C} & 5.003562 & .780092 & .184700 \\ \mathrm{H} & 5.783408 & .019518 & .191846 \\ \mathrm{H} & 5.161148 & 1.490661 & -.629862 \\ \mathrm{H} & 4.978322 & 1.318727 & 1.134703 \\ \mathrm{C} & -2.230868 & .967236 & -.270691 \\ \mathrm{H} & -3.175999 & .443471 & -.390729 \\ \mathrm{~N} & -2.412330 & 2.264337 & .004575 \\ \mathrm{C} & -3.755299 & 2.847111 & -.109148 \\ \mathrm{H} & -3.778448 & 3.588824 & -.915378 \\ \mathrm{H} & -4.487269 & 2.068700 & -.330390 \\ \mathrm{H} & -4.027588 & 3.339610 & .829732 \\ \mathrm{C} & -1.356939 & 3.218139 & .357139 \\ \mathrm{H} & -1.818919 & 4.061695 & .873695 \\ \mathrm{H} & -.629681 & 2.757369 & 1.028229 \\ \mathrm{H} & -.841132 & 3.599178 & -.531597 \\ \mathrm{O} & 2.025777 & -2.019138 & .846108 \\ \mathrm{H} & 2.135801 & -2.975048 & .702387\end{array}$

\section{5 (Enamin_reactant) $\mathrm{HF}=-901.6891116$}

\begin{tabular}{|c|c|c|c|}
\hline C & -1.402050 & -.131539 & -.246731 \\
\hline $\mathrm{C}$ & -.161938 & -.911920 & -.284940 \\
\hline $\mathrm{C}$ & 1.097533 & -.538231 & .036875 \\
\hline $\mathrm{C}$ & 1.479463 & .790938 & .615790 \\
\hline $\mathrm{C}$ & 1.287191 & .994371 & 1.935551 \\
\hline $\mathrm{C}$ & 2.136050 & -1.613262 & -.123925 \\
\hline $\mathrm{C}$ & -1.304102 & 1.277741 & -.374750 \\
\hline $\mathrm{O}$ & 3.356291 & -1.190554 & .245604 \\
\hline $\mathrm{O}$ & 1.899645 & -2.737746 & -.523544 \\
\hline
\end{tabular}




\begin{tabular}{|c|c|c|c|}
\hline $\mathrm{H}$ & -.247126 & -1.932670 & -.644008 \\
\hline $\mathrm{H}$ & 1.570939 & 1.906036 & 2.446826 \\
\hline $\mathrm{H}$ & .868175 & .203430 & 2.546101 \\
\hline $\mathrm{N}$ & 1.918612 & 1.775205 & -.289952 \\
\hline $\mathrm{C}$ & 2.382856 & 3.028663 & .296754 \\
\hline $\mathrm{H}$ & 1.590782 & 3.475353 & .906281 \\
\hline $\mathrm{H}$ & 3.270827 & 2.893048 & .933165 \\
\hline $\mathrm{H}$ & 2.633521 & 3.725451 & -.506831 \\
\hline $\mathrm{C}$ & 2.692777 & 1.361406 & -1.465448 \\
\hline $\mathrm{H}$ & 2.211204 & .512080 & -1.955177 \\
\hline $\mathrm{H}$ & 2.717964 & 2.191284 & -2.177309 \\
\hline $\mathrm{H}$ & 3.724162 & 1.079387 & -1.214028 \\
\hline $\mathrm{C}$ & 4.410192 & -2.172234 & .152904 \\
\hline $\mathrm{H}$ & 5.312180 & -1.659129 & .484359 \\
\hline $\mathrm{H}$ & 4.189428 & -3.024199 & .799887 \\
\hline $\mathrm{H}$ & 4.515416 & -2.520252 & -.877322 \\
\hline $\mathrm{H}$ & -.354811 & 1.658490 & -.747275 \\
\hline $\mathrm{N}$ & -2.183615 & 2.230359 & -.097255 \\
\hline $\mathrm{C}$ & -3.433168 & 2.052600 & .648667 \\
\hline $\mathrm{H}$ & -3.375198 & 1.152330 & 1.262649 \\
\hline $\mathrm{H}$ & -3.568924 & 2.919413 & 1.301179 \\
\hline $\mathrm{H}$ & -4.297402 & 1.991474 & -.021375 \\
\hline $\mathrm{C}$ & -1.891451 & 3.621375 & -.473912 \\
\hline $\mathrm{H}$ & -1.883297 & 4.250379 & .421995 \\
\hline $\mathrm{H}$ & -.920834 & 3.675676 & -.967368 \\
\hline $\mathrm{H}$ & -2.662906 & 3.989937 & -1.157888 \\
\hline $\mathrm{C}$ & -2.663517 & -.757582 & -.295441 \\
\hline $\mathrm{H}$ & -3.499919 & -.165144 & -.652484 \\
\hline $\mathrm{N}$ & -3.001924 & -2.017359 & -.033261 \\
\hline $\mathrm{C}$ & -2.161011 & -2.994623 & .671541 \\
\hline $\mathrm{H}$ & -2.804176 & -3.565077 & 1.347662 \\
\hline $\mathrm{H}$ & -1.686346 & -3.689628 & -.028456 \\
\hline $\mathrm{H}$ & -1.395006 & -2.485479 & 1.254164 \\
\hline
\end{tabular}


C

$\mathrm{H}$

$\mathrm{H}$

$\mathrm{H}$ $\begin{array}{lll}-4.322354 & -2.524054 & -.426813\end{array}$

$\begin{array}{lll}-4.901467 & -1.735438 & -.909619\end{array}$

$-4.204618-3.354796-1.130727$

$\begin{array}{lll}-4.860401 & -2.884878 & .455657\end{array}$

\section{TS-56}

C

C

C

C

C

C

$\mathrm{H}$

H

H

N

C

H

H

H

C

H

H

H

H

$\mathrm{N}$

C

O

O

C

H $\begin{array}{lll}1.560731 & 1.076742 & -.986111\end{array}$

$\begin{array}{llll}1.371006 & -.294439 & -.508114\end{array}$

$\begin{array}{llll}.080791 & -.946376 & -.609111\end{array}$

$\begin{array}{llll}-1.149916 & -.382663 & -.461438\end{array}$

$\begin{array}{llll}-1.300468 & 1.064241 & -.194353\end{array}$

$\begin{array}{lll}-.775944 & 1.933664 & -1.134491\end{array}$

$\begin{array}{lll}.070244 & -2.017838 & -.780080\end{array}$

$\begin{array}{llll}-.718080 & 1.592721 & -2.159434\end{array}$

$\begin{array}{llll}1.361231 & 1.283731 & -2.028345\end{array}$

$\begin{array}{llll}2.383745 & 1.954687 & -.407172\end{array}$

$\begin{array}{lll}2.633602 & 1.998251 & 1.036298\end{array}$

$\begin{array}{lll}3.707879 & 1.956314 & 1.241449\end{array}$

$\begin{array}{lll}2.241923 & 2.941208 & 1.437609\end{array}$

$2.137354 \quad 1.163134 \quad 1.527654$

$2.766513 \quad 3.174644 \quad-1.122205$

$\begin{array}{llll}2.643732 & 3.034610 & -2.196997\end{array}$

$\begin{array}{llll}2.146582 & 4.017609 & -.791480\end{array}$

$\begin{array}{llll}3.814786 & 3.406657 & -.913865\end{array}$

$\begin{array}{llll}-.792298 & 3.009523 & -.991750\end{array}$

$\begin{array}{lll}-1.859439 & 1.472110 & .974302\end{array}$

$\begin{array}{llll}-2.321356 & -1.305731 & -.563199\end{array}$

$\begin{array}{llll}-3.492956 & -.650800 & -.458148\end{array}$

$\begin{array}{lll}-2.227679 & -2.505804 & -.735644\end{array}$

$\begin{array}{llll}-4.674339 & -1.470750 & -.586291\end{array}$

$\begin{array}{llll}-5.514303 & -.784917 & -.481222\end{array}$ 


$\begin{array}{llll}\mathrm{H} & -4.691379 & -1.957053 & -1.564152 \\ \mathrm{H} & -4.694716 & -2.234213 & .194960 \\ \mathrm{C} & -2.130923 & .580712 & 2.097313 \\ \mathrm{H} & -1.763303 & 1.034221 & 3.024991 \\ \mathrm{H} & -3.206324 & .394700 & 2.200257 \\ \mathrm{H} & -1.622284 & -.373210 & 1.957378 \\ \mathrm{C} & -2.223416 & 2.870054 & 1.177135 \\ \mathrm{H} & -2.997761 & 2.928286 & 1.945709 \\ \mathrm{H} & -1.366839 & 3.475739 & 1.505396 \\ \mathrm{H} & -2.622659 & 3.294973 & .252382 \\ \mathrm{C} & 2.546148 & -.953003 & -.141205 \\ \mathrm{H} & 3.471573 & -.404595 & -.300237 \\ \mathrm{~N} & 2.760454 & -2.173778 & .370672 \\ \mathrm{C} & 4.119972 & -2.720953 & .402379 \\ \mathrm{H} & 4.199237 & -3.584628 & -.267998 \\ \mathrm{H} & 4.838236 & -1.964772 & .080803 \\ \mathrm{H} & 4.367927 & -3.044044 & 1.418905 \\ \mathrm{C} & 1.724224 & -3.080387 & .868186 \\ \mathrm{H} & 2.145054 & -3.659033 & 1.695200 \\ \mathrm{H} & .869337 & -2.512163 & 1.235315 \\ \mathrm{H} & 1.386861 & -3.779284 & .094513\end{array}$

\section{References}

${ }^{1}$ Krasnaya, Zh. A.; Stytsenko, T. S.; Bogdanov, V. S.; Daeva, E. D. Izvestiya Akademii Nauk SSSR, Seriya Khimicheskaya 1985, 7,1604-12. (in Russian).

${ }^{2}$ Smith; Knerr J. Am. Chem. Soc. 1886, 8, 99.

${ }^{3}$ Arnone, C.; Consiglio, G.; Frenna, V.; Spinelli, D. J. Org. Chem. 1997, 62, 3093.

${ }^{4}$ A 1:1 mixture of benzaldehyde/benzaldehyde- $D$ in 10 fold excess has been used to determine the isotope effect in the addition of silylenol ethers see: Myers, A. G.; Widdowson, K. L.; Kukkola, P. J. J. Am. Chem. Soc. 1992, 114, 2765. 